\title{
La coopérative minière: instrument de l'ingérence étatique dans la liberté d'association des exploitants miniers artisanaux en République Démocratique du Congo?
}

\author{
Par Patient LWANGO MIRINDI ${ }^{1}$
}

\section{INTRODUCTION}

En République Démocratique du Congo (RDC), la Constitution prévoit à son article 58 que tous les Congolais ont le droit de jouir des richesses nationales et que celles-ci doivent être redistribuées équitablement par l'Etat. ${ }^{2}$ Le droit de jouissance garanti aux Congolais et l'obligation de redistribution à charge de l'Etat impliquent de la part de ce dernier, en vertu de sa souveraineté sur ses ressources naturelles, ${ }^{3}$ qu'il organise les conditions d'accès aux richesses naturelles se trouvant sur le territoire national.

1 Patient LWANGO MIRINDI est doctorant à la Vrije Universiteit Brussel (VUB), chercheur à l'Université Catholique de Bukavu et avocat près la Cour d'appel de Bukavu.

2 L'article 58 de la Constitution de la RDC est ainsi libellé:

« Tous les Congolais ont le droit de jouir des richesses nationales.

L'Etat a le devoir de les redistribuer équitablement et de garantir le droit au développement ».

3 En ce qui concerne la souveraineté de la RDC sur ses ressources naturelles, l'article 9 de la Constitution stipule ce qui suit:

«L'Etat exerce une souveraineté permanente notamment sur le sol, le sous-sol, les eaux et les forêts, sur les espaces aérien, fluvial, lacustre et maritime congolais ainsi que sur la mer territoriale congolaise et sur le plateau continental.

Les modalités de gestion et de concession du domaine de l'Etat visé à l'alinéa précédent sont déterminées par la loi ».

De son côté, l'article 3 alinéa 1 de la loi n007-2002 du 11 juillet 2002 portant Code minier de la RDC, parlant du droit de propriété de l'Etat sur les substances minérales se trouvant sur le territoire national, précise que :

«Les gîtes des substances minérales, y compris les gîtes artificiels, les eaux souterraines et les gîtes géothermiques se trouvant sur la surface du sol ou renfermés dans le sous-sol ou dans les cours d'eaux du Territoire National sont la propriété exclusive inaliénable et imprescriptible de l'Etat ». 
Parmi ces richesses, on trouve les minerais ${ }^{4}$ dont l'exploitation est régie par la loi $\mathrm{n}$ ${ }^{\circ} 007-2002$ du 11 juillet 2002 portant Code minier de la RDC (abrégée ici par Code minier), laquelle contient des dispositions relatives à l'activité des exploitants miniers artisanaux opérant soit de manière individuelle, soit en groupes appelés coopératives minières. ${ }^{5}$

Dans la pratique, l'Etat congolais manifeste une préférence pour le groupement d'exploitants miniers artisanaux et a tendance à obliger à ces exploitants de se regrouper. Notre hypothèse de départ est que le groupement d'exploitants artisanaux, tel qu'envisagé par le Code minier est conforme à la liberté d'association prévue par la Constitution de la RDC et les instruments internationaux des droits de l'homme ratifiés par ce pays. ${ }^{6}$ Il convient ainsi de s'interroger sur la pratique étatique, pour en examiner la conformité au droit interne et au droit international par rapport à la liberté d'association.

L'approche suivie consistera d'abord à examiner le droit minier congolais dans ses dispositions relatives à l'exploitation minière artisanale. Ensuite, la pratique de l'Etat congolais ${ }^{7}$ sera confrontée au contenu de la liberté d'association et aux conditions d'admissibilité

4 Les minerais de la RDC peuvent être classés en six catégories, à savoir: le groupe du cuivre (cuivre, cobalt, uranium, zinc, plomb, cadmium, germanium) se trouvant majoritairement au Katanga; le groupe de l'étain (étain, wolfram, colombo-tantalite, béryl, monazite) localisé dans la Province Orientale, le Maniema, le Nord-Kivu, le Sud-Kivu et le Nord-Katanga; le groupe chrome, nickel, niobium, diamant localisés principalement dans les deux Kasaï; les métaux précieux (l'or exploité dans la Province Orientale, le Maniema, le Nord-Kivu, le Sud-Kivu, les deux Kasaï, le Katanga et le Bas-Congo; l'argent se trouvant au Katanga, en Province Orientale, au Maniema, au Nord-Kivu et au Sud-Kivu; le platine dans les Kasaï et en Province Orientale); le fer et le manganèse dans la Province Orientale, les Kasaï et le Katanga; les combustibles minéraux (charbon au Katanga, schistes bitumineux en Province Orientale et au Bas-Congo, pétrole et gaz dans le Bas-Congo et dans la vallée du Graben de l'Ituri au lac Tanganyika. Voir à cet effet MAZALTO (Marie), Gouvernance du secteur minier et enjeux de développement en République Démocratique du Congo, Thèse de doctorat en sociologie, Montréal, Université du Québec, 2010, pp.67-68; Ipis et International Alert, La complexité de la gestion des ressources dans un contexte de fragilité de l'Etat : une analyse du secteur minier dans l'arrière-pays du Kivu, Steven Spittaels (éd.), 2010, pp.12-13, 28-31, 46-48.

5 Comme le souligne Triest, l'exploitation artisanale des minerais " octroie des moyens de survie à ceux qui tentent d'éviter de sombrer dans la pauvreté absolue ou d'en sortir. Sans cet ultime filet, la situation sociale à l'Est du Congo serait encore plus tendue qu'elle ne l'est actuellement ». Voir Triest (Frédéric), Justice et Paix. Analyses 2012. Le secteur minier artisanal à l'Est de la RDC: état des lieux et perspectives, Bruxelles, Commission Justice et Paix Belgique francophone, Mai 2012, p.3.

6 Les instruments internationaux des droits de l'homme ratifiés par la RDC font partie de l'ordonnancement juridique interne du pays, en vertu des dispositions de l'article 215 de la Constitution qui dispose: «Les traités et accords internationaux régulièrement conclus ont, dès leur publication, une autorité supérieure à celle des lois, sous réserve pour chaque traité ou accord, de son application par l'autre partie».

7 Sont ainsi analysés, en plus des déclarations émanant de différentes autorités publiques, les arrêtés

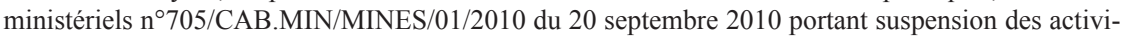
tés minières dans les Provinces du Maniema, du Nord-Kivu et du Sud-Kivu, nº706/CAB.MIN/ MINES/01/2010 du 20 septembre 2010 portant mesures d'encadrement de l'Arrêté ministériel $n$

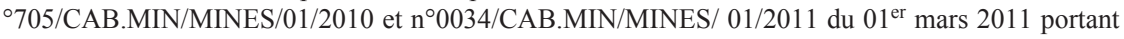
levée de la mesure de suspension des activités minières dans les trois provinces susvisées. Les arrê- 
des restrictions à cette liberté, telles qu'elles découlent d'une part des instruments juridiques internes (Constitution, Code minier, Décret n $038 / 2003$ du 26 mars 2003 portant Règlement minier de la $\mathrm{RDC}^{8}$ ) et d'autre part des instruments internationaux pertinents relatifs aux droits de l'homme ratifiés par la RDC à savoir le Pacte international relatif aux droits civils et politiques (PIDCP), le Pacte international relatif aux droits économiques, sociaux et culturels (PIDESC) $)^{9}$ et la Charte africaine des droits de 1 'homme et des peuples. ${ }^{10}$ Pour préciser le contenu de la liberté d'association, des décisions du Comité des droits de l'homme des Nations Unies (CDH), ainsi que celles de la Cour et de la Commission africaines des droits de l'homme et des peuples seront examinées, de même que la doctrine y relative.

La contribution est subdivisée en deux grands points. Le premier montre comment, d'une simple faculté, le regroupement des exploitants miniers artisanaux est en phase de devenir une obligation. Le second cherche à savoir si cette obligation est compatible avec la liberté d'association.

\section{LE REGROUPEMENT DES EXPLOITANTS MINIERS ARTISANAUX: D'UNE FACULTE A UNE OBLIGATION}

Trois formes d'exploitation minière sont prévues en RDC, à savoir l'exploitation industrielle, l'exploitation à petite échelle et l'exploitation artisanale. C'est cette dernière qui est abordée ici, parce que l'analyse porte sur les groupements d'exploitants miniers artisanaux.

tés ministériels susmentionnés ont fait l'objet d'études qui se sont intéressées à leur impact socioéconomique (voir par exemple GEENEN (Sara), KAMUNDALA (Gabriel) et IRAGI (Francine), " Le pari qui paralysait : La suspension des activités minières artisanales au Sud-Kivu ", in L'Afrique des Grands Lacs. Annuaire 2010-2011, Paris, L'Harmattan, pp.161-183; Pole Institute, Réouverture des activités minières à l'Est de la RD Congo: enjeux et défis, Goma, Avril 2011, http:/ /www.pole-institute.org/site\%20web/echos/echo148. htm, consulté le 06/08/2013). La présente contribution enrichit la réflexion ainsi amorcée par l'analyse des questions juridiques que soulève une des décisions contenues dans lesdits arrêtés, à savoir l'obligation pour les exploitants artisanaux de se regrouper en coopératives. Elle s'avère d'actualité dans la mesure où des réflexions sont en cours en RDC en vue d'une révision du Code minier.

8 Ici abrégé par Règlement minier.

9 Les deux Pactes internationaux ont été ratifiés par la RDC le $1^{\text {er }}$ novembre 1976 (Voir Journal Officiel de la RDC, Instruments internationaux relatifs aux Droits de l'Homme ratifiés par la République Démocratique du Congo, numéro spécial 40ème année, 9 avril 1999, pp.12, 21).

10 La Charte africaine des droits de l'homme et des peuples, ici abrégée par Charte africaine, a été ratifiée par la RDC le 20 juillet 1987. Voir Union Africaine, Liste des pays qui ont signé, ratifiél adhéré. Charte africaine des droits de l'homme et des peuples, http://www.africa-union.org/root/a u/Documents/Treaties/List/African\%20Charter\%20on\%20Human\%2 0and20Peoples\%20Rights.p df, consulté le 26/08/2013. 


\section{L'exploitation minière artisanale dans le Code minier}

D'après l'article $1^{\text {er }}$ alinéa 21 du Code minier, l'exploitation artisanale est l'activité par laquelle une personne physique de nationalité congolaise se livre, dans une zone d'exploitation artisanale délimitée en surface et en profondeur jusqu'à trente mètres au maximum, à extraire et à concentrer des substances minérales en utilisant des outils, des méthodes et des procédés non industriels.

Cette forme d'exploitation est réservée aux personnes physiques majeures de nationalité congolaise (article $26 \mathrm{du}$ Code minier), pourvu que ces personnes ne soient pas frappées par les causes d'incapacité énumérées par le Code minier. ${ }^{11}$

Par ailleurs, l'exploitation artisanale ne doit être réalisée que dans une aire géographique délimitée par l'autorité publique et appelée « zone d'exploitation artisanale » (ZEA).

\section{a. L'exploitation artisanale doit être effectuée dans une ZEA}

La ZEA est instituée par le Ministre national des Mines, après avis de la Direction des Mines et du Gouverneur de la province concernée. ${ }^{12}$ La personne qui désire exploiter de manière artisanale des minerais doit être détentrice d'une carte d'exploitant artisanal délivrée annuellement par le Chef de division provinciale des mines (article 111 du Code minier) et qui indique la ZEA dans laquelle l'activité artisanale sera effectuée. ${ }^{13}$

11 L'article 27 du Code minier, intitulé « Des personnes non éligibles », stipule ce qui suit:

« Ne sont pas éligibles pour solliciter et obtenir les droits miniers et/ou de carrières, les cartes d'exploitant artisanal, de négociants ainsi que l'agrément au titre de comptoir d'achat et de vente des substances minérales d'exploitation artisanale:

a) les agents et fonctionnaires de l'Etat, les Magistrats, les membres des Forces Armées, la Police et les Services de Sécurité, les employés des organismes publics habilités à procéder aux opérations minières.

Toutefois, cette incompatibilité ne concerne pas leur prise de participation dans le capital des sociétés minières;

b) toute personne frappée d'incapacité juridique prévue à l'article 215 de la loi nº87-010 du 01 août 1987 portant Code de la Famille;

c) toute personne frappée d'interdiction notamment:

- la personne condamnée par un jugement coulé en force de chose jugée pour des infractions à la législation minière et de carrières ou à celles se rapportant aux activités économiques de ses droits miniers et de carrières et de ses sociétés affiliées et ce, pendant dix ans;

- la personne à laquelle la carte d'exploitation artisanale ou de négociant a été retirée et ce, pendant trois ans;

- la personne à laquelle l'agrément au titre des comptoirs d'achat et de vente des substances minérales d'exploitation artisanale a été retirée et ce, pendant cinq ans ».

12 Voir l'article 109 du Code minier.

13 Aux termes de l'article 111 du Code minier, seuls les détenteurs des cartes d'exploitant artisanal en cours de validité pour la ZEA concernée sont autorisés à exploiter l'or, le diamant ou toute autre substance minérale qui est exploitable artisanalement. 
Cependant, dans la pratique, de nombreux exploitants miniers artisanaux ne sont pas détenteurs de cartes d'exploitants artisanaux. Par exemple, au Nord-Kivu et au Sud-Kivu, « des dizaines de milliers de personnes, dont des enfants, travaillent comme creuseurs artisanaux ... Impossible d'en connaître le nombre exact car ils ne sont pas inscrits». ${ }^{14}$

La présence dans les zones minières d'exploitants artisanaux non enregistrés par l'Etat s'explique non seulement par la modicité des revenus de ces exploitants, qui ne leur permettent pas de solliciter et obtenir les cartes d'exploitants artisanaux. En effet, « ces revenus journaliers ne dépassant pas 10 \$ US pour la plupart d'entre eux, sont dépensés sur place pour se nourrir et s'acheter des produits finis disponibles sur le site minier ». ${ }^{15}$ Elle s'explique aussi par le fait que certains exploitants artisanaux ne veulent tout simplement pas être identifiés ${ }^{16}$ pour ne pas payer les multiples frais exigés par les services de l'Etat. ${ }^{17}$

14 Global Witness, Face à un fusil, que peut- on faire? La guerre et la militarisation du secteur minier dans l'est du Congo, Rapport, juillet 2009, p.27.

15 Pole Institute, Réouverture des activités minières à l'Est de la RD Congo : enjeux et défis, Goma, Avril 2011, http://www.pole-institute.org/site\%20web/echos/echo148.htm, consulté le 06/08/2013.

16 Banque mondiale, République Démocratique du Congo. La bonne gouvernance dans le secteur minier comme facteur de croissance, Rapport $\mathrm{N}^{\circ}$ 43402-ZR, Mai 2008, p.65.

17 Pour donner une idée des taxes exigées, des exploitants artisanaux du Sud-Kivu, réunis dans un atelier par l'ONG Aprodeped à Bukavu en 2010 avaient inventorié plusieurs frais à payer pour la plupart annuellement, et ce en dehors des impôts et taxes reconnus par le Code et le Règlement miniers. Ces frais payés sur le terrain, qui diffèrent d'un site d'exploitation artisanale à un autre, sont souvent exigés sans délivrance de quittance (ce qui pose un problème de contrôle de ces recettes par l'Etat). A titre d'exemple, pour le site de Kamole dans le Territoire d'Idjwi, le Chef d'antenne des Mines exigeait de payer $35 \$$ US pour la carte de creuseur (à obtenir par la personne qui déclare détenir le droit d'exploiter la mine). En plus, pour expédier la production vers le lieu de vente il fallait payer 5 \$US pour une carte de convoyeur et 5 \$US pour une attestation de transfert. Sur le même site, le Chef d'un autre service public chargé de l'encadrement des exploitants artisanaux (le Service d'assistance et d'encadrement du small scale mining ou SAESSCAM) exigeait à l'exploitant d'une mine de payer la somme de $2 \$$ par travailleur/creuseur employé, ainsi que $1 \%$ de la production obtenue. Il demandait en outre aux négociants (qui achètent les produits miniers artisanaux pour les revendre aux comptoirs) de payer $1 \%$ du produit acheté. Toujours sur le même site, l'autorité coutumière faisait payer $10 \$$ US pour chaque mine et $10 \%$ de la production de la mine, ainsi que 2\$US par creuseur employé). Pour le site de Kasika dans le territoire de Mwenga, le chef d'antenne des mines exigeait de payer $25 \$$ US pour la carte de creuseur, $10 \$$ US pour la fiche d'identification, 15\$US pour l'embarcation de la production; tandis que le SAESSCAM et un autre service public, la DGRAD, exigeaient chacun 20\$US pour la carte de creuseur. Enfin, à l'autorité coutumière il fallait payer 20\$US comme redevance coutumière. Dans des sites du territoire de Walungu, il était exigé de payer 20\$US ou 25\$US pour obtenir la carte de creuseur, 1 \$ US au SAESSCAM pour la carte de recensement et 10\$US à la coopérative COMALU pour la carte de membre. Au site de Kamituga dans le territoire de Mwenga, il fallait payer : 25\$US pour la carte de creuseur, $15 \$$ US pour une fiche technique de la mine, 10\$US pour la fiche d'identification, 10 SUS pour l'autorisation d'ouverture de la mine, 10\$US pour l'enregistrement, 10\$US par mois par mine au SAESSCAM, 75\$US au service public de l'énergie et hydrocarbures comme taxe sur motopompe et 500\$US par an en cas d'utilisation d'un générateur électrique; 40\$US à 45\$US à l'autorité coutumière. Au site de Kalehe dans le territoire du même nom, il était exigé 50\$US à $75 \$$ US pour la carte de creuseur, 30\$US pour l'attestation de transport, un pourcentage de la production pour l'entité administrative (le territoire) et un autre pourcentage pour l'autorité coutu- 
Par ailleurs, de nombreux exploitants artisanaux opèrent fréquemment dans des concessions déjà couvertes par des titres miniers, alors qu'aux termes de l'article 109 du Code minier, un périmètre minier faisant l'objet d'un titre minier en cours de validité ne peut pas être transformé en zone d'exploitation artisanale. A titre d'exemple « au Katanga, des milliers d'exploitants miniers artisanaux ont envahi des concessions appartenant à la GECAMINES et à d'autres sociétés privées $»{ }^{18}$ De même, au Maniema, dans le Nord-Kivu et le Sud-Kivu, les exploitants artisanaux œuvrent dans des concessions minières attribuées jadis à la société Sominki et aujourd'hui à la société Banro. ${ }^{19}$

Le regroupement des exploitants miniers artisanaux a été, comme nous allons le voir plus loin, considéré par l'Etat comme l'une des solutions pour arriver à identifier et contrôler lesdits exploitants. Pour l'instant, il importe de relever que c'est la détention de la carte d'exploitant artisanal qui donne le droit d'exploiter de manière artisanale des minerais, individuellement ou en groupe.

mière, 5 \$US pour une fiche d'identification. Il fallait en outre payer au SAESSCAM $3 \$$ US pour la même fiche d'identification de l'exploitant de la mine, outre 10\$US pour l'identification de la mine et une montant forfaitaire variable au service chargé de la lutte contre la fraude. Voir Aprodeped, Rapport du séminaire de formation sur les droits des communautés locales dans l'exploitation des ressources naturelles en République Démocratique du Congo, Bukavu du 12 au 14 février 2010, pp.34-37. Cette longue liste de frais montre que l'exploitation artisanale procure des recettes aux services publics et que l'une des questions à résoudre est celle de savoir comment drainer ces recettes vers le Trésor public. Comme relevé plus loin, le groupement d'exploitants artisanaux est l'une des solutions envisagées par l'Etat.

18 Banque mondiale, op.cit., p.63.

19 Ministère des Mines de la RDC-BGR, Rapport de l'atelier de planification du projet BGR-MDM : certification des substances minérales, Bukavu, mars 2010, pp.34-35; De Failly (Didier), « L'économie du Sud-Kivu 1990-2000 : Mutations profondes cachées par une panne», in L'Afrique des Grands Lacs. Annuaire 1999-2000, Paris, L'Harmattan, 2000, p.182; De Failly (Didier), « Coltan : Pour comprendre... ", in L'Afrique des Grands Lacs. Annuaire 2000-2001, Paris, L'Harmattan, 2001, p. 281. La mine de Bisie dans le territoire de Walikale au Nord-Kivu est un cas supplémentaire du chaos qui peut régner sur un site minier. Sur ce site, un exploitant titulaire d'un droit minier d'exploration (voulu exclusif par le Code minier) se disputait le droit d'exploitation avec deux coopératives minières. Dans le même temps, les deux coopératives étaient concurrentes et prétendaient chacune avoir le droit exclusif d'exploiter la mine. Elles avaient soumis leur litige à la justice, mais n'hésitaient pas à recourir à des groupes armés et parfois à l'armée nationale pour régler leur différend. A ce sujet, lire notamment Global Witness, op.cit, p.36. Fermée pendant un certain temps sur décision du Gouvernement central, la mine de Bisie a été rouverte récemment, sur décision du Ministre national des mines et après un plaidoyer du Gouverneur de la Province du NordKivu, comme le renseigne le site web de ce dernier, dans le document intitulé Ouverture du site minier de Bisie en territoire de Walikale, http://www.julienpaluku.com/index.php/info-du-nord-ki vu/51-nord-kivu/5008-ouverture-du-site-minier-de-bisie-en-territoire-de-walikale, consulté le 14/10/2013. Il reste à espérer que l'Etat a pris les dispositions nécessaires pour résoudre les problèmes de sécurité et les conflits concernant les droits d'exploitation dans cette mine. Au moins, l'exemple montre que le regroupement des exploitants artisanaux ne constitue pas, à lui seul, la solution à tous les problèmes des exploitants artisanaux. 
La coopérative minière: instrument de l'ingérence étatique dans la liberté d'association des exploitants miniers artisanaux en République Démocratique du Congo?

\section{b. L'exploitation artisanale (individuelle ou collective)}

Les exploitants artisanaux qui souhaitent améliorer leurs méthodes de travail et leur rendement peuvent se regrouper et former une personne morale appelée par le Code minier « groupement des exploitants artisanaux $» .^{20}$

A la différence de l'exploitant artisanal individuel, le groupement d'exploitants artisanaux a, à l'instar de l'exploitant minier industriel, le droit d'obtenir des droits miniers exclusifs (permis de recherche, permis d'exploitation, permis d'exploitation des rejets de mine) dans la ZEA concernée par son activité (article 109 du Code minier). Ce qui assure une protection juridique aux droits acquis par le groupement d'exploitants artisanaux.

En outre, en cas de fermeture d'une ZEA due au fait que les facteurs ayant justifié sa création ont cessé d'exister ou qu'un nouveau gisement ne relevant pas de l'exploitation artisanale vient à être découvert, le groupement d'exploitants artisanaux travaillant dans la ZEA concernée dispose, en vertu de l'article 110 du Code minier, d'un droit de préemption pour solliciter un permis en vue d'une exploitation industrielle ou à petite échelle. ${ }^{21}$

Le groupement d'exploitants artisanaux prend la forme d'une coopérative minière. ${ }^{22} \mathrm{En}$ d'autres termes, les exploitants artisanaux demeurent libres de se regrouper ou non; mais pour ceux qui auront choisi de se regrouper, la personne morale à créer devra prendre la forme d'une coopérative minière. ${ }^{23}$ Ainsi, malgré les avantages que peuvent présenter, pour

20 Code minier, articles 109 et 110 .

21 L'exploitation minière à petite échelle est « toute activité par laquelle une personne se livre à une exploitation de petite taille et permanente, exigeant un minimum d'installations fixes en utilisant des procédés semi-industriels ou industriels, après la mise en évidence d'un gisement » (article 1 alinéa 22 du Code minier).

Ce type d'exploitation « présente notamment les caractéristiques suivantes :

a) le montant de l'investissement requis varie entre USD 100.000 et USD 2.000.000;

b) les réserves exploitables ne dépassent pas une durée de vie de dix ans sous réserve des dispositions de l'article 101 alinéa 2 du Code Minier;

c) les opérations d'extraction, de transport et de traitement de minerais sont suffisamment mécanisées.

Le Ministre peut modifier les caractéristiques de l'exploitation minière à petite échelle par voie d'arrêté après avis de la Direction des Mines » (article 204 du Règlement minier).

22 Voir en effet l'article 234 du Règlement minier qui stipule:

« Les groupements d'exploitants artisanaux qui désirent procéder à la recherche de substances minérales classées en mines à l'intérieur de la zone d'exploitation artisanale à l'aide de procédés industriels ou semi-industriels sont tenus de se constituer en coopérative et solliciter auprès du Ministre ayant les Mines dans ses attributions l'agrément au titre de coopérative minière.

Pour être agréée, la coopérative doit être composée de personnes détentrices de cartes d'exploitant artisanal valables pour la zone d'exploitation artisanale à l'intérieur de laquelle se trouve le périmètre sur lequel la coopérative souhaite obtenir un Permis de Recherches. En plus, la coopérative doit introduire une demande d'agrément au titre de coopérative minière qui remplit les conditions précisées à l'article suivant $»$.

23 En droit congolais (sous réserve des modifications devant découler de l'adhésion de la RDC à l'Organisation pour l'Harmonisation en Afrique du Droit des Affaires) il existe deux formes de 
les exploitants artisanaux, leur regroupement en coopérative, le Code minier ne fait pas d'un tel regroupement un préalable pour l'exercice de l'activité minière dans une ZEA, le préalable étant plutôt la détention d'une carte d'exploitant artisanal pour l'exploitant individuel.

Nous pouvons mentionner, comme preuve supplémentaire de la liberté pour les exploitants artisanaux de constituer ou non des personnes morales, le fait que dans le Code minier on trouve des dispositions distinctes qui s'appliquent aux exploitants artisanaux œuvrant de manière individuelle ${ }^{24}$ et aux groupements d'exploitants artisanaux. ${ }^{25}$

Par ailleurs, même le Règlement minier préserve l'activité individuelle des exploitants artisanaux, car il précise que la demande d'agrément au titre de coopérative minière doit comprendre, entre autres documents, la preuve que l'adhésion au groupement d'exploitants artisanaux a été proposée à tous les exploitants artisanaux travaillant dans la ZEA (article 235 alinéa f), la preuve de la publication de l'avis d'adhésion à la Division Provinciale des Mines pendant six mois (article 235 alinéa f), la preuve du contact personnel pris avec les exploitants artisanaux de la ZEA (article 235 alinéa f), la preuve des signatures des exploitants dans la fiche d'adhésion (article 235 alinéa f), la preuve que les conditions d'adhésion au groupement ne sont pas prohibitives (article 235 alinéa g).

Or, en obligeant à la coopérative candidate à l'agrément de prouver que l'adhésion en son sein a été proposée aux exploitants artisanaux et que les conditions d'adhésion n'ont pas été prohibitives, le Règlement minier protège les droits des exploitants miniers présents dans la ZEA sur laquelle la coopérative cherche à obtenir un droit minier exclusif : la consultation de ces exploitants individuels par la coopérative permet d'éviter que les individus non membres de la coopérative ne soient surpris par une expulsion de la ZEA concernée.

coopératives, organisées toutes les deux par des textes datant de l'époque coloniale, à savoir: d'une part la société coopérative régie par le Décret du 23 mars 1921 (ce texte organisait le fonctionnement des coopératives commerciales créées à l'époque par les colons); d'autre part l'association coopérative (anciennement appelée coopérative indigène) régie par le Décret du 24 mars 1956 et dont le statut emprunte aux règles des sociétés commerciales et des associations sans but lucratif. Ni le Code, ni le Règlement miniers ne précisent laquelle de ces deux formes prendra le groupement d'exploitants miniers artisanaux. La pratique montre que ce sera la forme de la coopérative prévue par le Décret du 24 mars 1956. Par exemple, dans l'arrêté ministériel N0942/CAB.MIN/ MINES/01/2012 du 24/12/2012 portant agrément de la Coopérative minière de développement du Katanga « COMIDEKAT » par le Ministre national des Mines, ce dernier se réfère au Décret du 24 mars 1956 et non pas à celui du 23 mars 1921 (Voir ledit arrêté dans le site du Ministère des mines, http://mines-rdc.cd/fr/documents/Arretes/A0942_2012.pdf, consulté le 14/10/2013). On notera que la coopérative du décret du 24 mars 1956 est agréée par le Gouverneur de Province, tandis que la coopérative minière est agréée par le Ministre national des Mines. Dans la Province du Sud-Kivu, les services publics provinciaux (Division provinciale des Mines et SAESSCAM) ont produit un statut-type de coopérative minière, auquel les exploitants artisanaux sont appelés à se référer pour préparer leur demande d'agrément à envoyer au Ministère national des mines.

24 Articles 111 et 112 du Code minier.

25 Notamment les articles 109 par. 4 et 110 par. 3 à 5 du Code minier. 
En outre, exiger la consultation des exploitants présents dans la ZEA sous-entend que ces exploitants demeurent libres d'adhérer ou non à la coopérative en formation ou à celle déjà formée, d'autant que le Règlement minier n'a pas indiqué que pour être agréé comme coopérative minière le groupement d'exploitants artisanaux doit prouver qu'il regroupe tous les exploitants artisanaux œuvrant dans la ZEA concernée.

Il convient de relever que la coopérative minière semble jouir de la faveur du Règlement minier par rapport aux exploitants individuels. En effet, le groupement candidat à l'agrément au titre de coopérative a comme seule obligation de solliciter l'adhésion des exploitants individuels. En conséquence, ce n'est qu'en démontrant qu'ils n'ont pas été consultés que les exploitants individuels non membres de la coopérative candidate à l'agrément pourraient s'opposer à cet agrément ou à l'obtention de droits miniers exclusifs par une telle coopérative. Autrement dit, si ladite coopérative démontre avoir consulté tous les exploitants individuels se trouvant dans une ZEA, elle obtiendra le droit minier sollicité, même si certains des exploitants consultés ont choisi de ne pas adhérer à la coopérative en formation. Dans pareille hypothèse, les exploitants pourtant munis de carte d'exploitant valide pour la ZEA concernée se trouveraient exclus de ladite zone, sans que le Code ou le Règlement miniers n'aient prévu une indemnisation ou compensation en leur faveur. ${ }^{26}$

Cependant, ni le Code ni le Règlement miniers n'imposent aux personnes physiques détentrices de la carte d'exploitant artisanal de se constituer en coopérative minière avant de procéder à l'exploitation minière artisanale. Ce qui est loin d'être le cas de la pratique des autorités publiques, laquelle manifeste une tendance à imposer aux exploitants artisanaux de se regrouper.

Comme démontré dans les développements qui suivent, cette tendance s'est cristallisée à travers les mesures de suspension de l'activité minière artisanale dans l'Est de la RDC de septembre 2010 à mars 2011.

26 Dans la pratique, il n'est pas évident que tous les exploitants individuels soient consultés, étant donné la manière dont les coopératives sont créées. Par exemple, citant le cas du Sud-Kivu, Kamundala relève que « le contexte de ... création de coopératives minières au Sud-Kivu reflète le caractère opportuniste de ses initiateurs. En effet, lors de la suspension des activités minières en septembre 2010, plusieurs recommandations ont été faites pour permettre la levée de la mesure de suspension, notamment pour le regroupement des creuseurs artisanaux au sein de coopératives minières. Plusieurs creuseurs, du moins les plus influents, ont constitué des dossiers pour leur coopérative. Ainsi la Division des mines s'est vite retrouvée avec plusieurs dossiers: en juin 2011, 46 coopératives avaient déjà été enregistrées pour 59.303 creuseurs. La précipitation dans laquelle ces coopératives se sont mises en place, parfois à l'initiative d'un individu qui coopte les autres, ne favorise pas la vulgarisation des principes de fonctionnement d'une coopérative, qui doivent être observés strictement». Voir KAMUNDALA BYEMBA (Gabriel), Exploitation minière industrielle versus exploitation minière artisanale au Sud-Kivu: Possibilités d'une cohabitation pacifique?, CEGEMI/UCB, Anvers, décembre 2012, p.14. 
2. La suspension de l'exploitation minière artisanale dans l'Est de la RDC de septembre 2010 à mars 2011 et son impact sur la liberté d'association des exploitants artisanaux

En visite dans la ville de Goma, capitale provinciale du Nord-Kivu, au début du mois de septembre 2010, le Président de la RDC décida de suspendre l'exploitation minière artisanale dans les provinces du Maniema, du Nord et du Sud-Kivu. Dans un communiqué en date du 11 septembre 2010, le Ministre national des Mines explique que le Président de la République a pris cette décision après avoir constaté :

" l'ampleur de l'exploitation minière dans cette partie du pays, laquelle résulte de fait des activités de groupes mafieux qui confortent, en dépit des efforts de stabilisation, l'insécurité récurrente; le paradoxe entre les richesses minières dont regorgent les provinces de l'Est et la pauvreté généralisée de leurs populations; l'implication manifeste de certaines autorités locales, provinciales et nationales tant civiles que militaires dans l'exploitation illégale et le commerce illicite des substances minérales $\gg .27$

La mesure de suspension sera complétée par l'arrêté ministériel n705/CAB.MIN/MINES/ 01/2010 du 20 septembre 2010 signé par le Ministre national des Mines et portant suspension des activités minières dans les provinces du Maniema, du Nord et du Sud-Kivu, lequel indiqua que la suspension ne concernait que l'exploitation artisanale, mais pas les titulaires de titres miniers en phase effective et régulière de développement et de construction (article 2 dudit arrêté). Les motifs de la décision furent de nouveau précisés par le Ministre.

a. Les motifs de la suspension

Dans son préambule, l'arrêté ministériel n05/CAB.MIN/MINES/01/2010 explique que la suspension a été décidée pour lutter contre le lien entre l'exploitation illégale et le commerce illicite des ressources minérales, la prolifération et le trafic d'armes par des groupes mafieux et armés, et l'insécurité récurrente dans les provinces concernées. Elle vise en outre à sauvegarder la souveraineté de l'Etat et à rétablir son autorité sur le sol et le soussol, à empêcher l'immixtion des agents et personnes étrangers aux services reconnus par le Code minier dans le circuit de l'exploitation et de la commercialisation des substances minérales et à lutter contre la fraude et la contrebande minières.

A partir des motifs énumérés ci-dessus, nous pouvons estimer que la décision de suspendre l'exploitation minière artisanale découlait de la volonté de la RDC de contrôler davantage cette exploitation. L'Etat congolais a ainsi exercé un des attributs de sa souveraine-

27 Voir le communiqué du Ministre des mines, publié sur le site de l'Association internationale des industriels de l'étain (ITRI), http://www.itri.co.uk/pooled/articles/BF_PARTART/view.asp?Q=BF _PARTART_310250, consulté le 03/11/2010. 
La coopérative minière: instrument de l'ingérence étatique dans la liberté d'association des exploitants miniers artisanaux en République Démocratique du Congo?

té territoriale, à savoir la plénitude de ses compétences sur son territoire, et ce en vertu de sa souveraineté permanente sur ses ressources naturelles et ses activités économiques. ${ }^{28}$

La levée de la suspension fut subordonnée, notamment, à l'obligation faite aux exploitants miniers artisanaux de se regrouper. Les coopératives minières existantes signèrent avec le Gouvernement un acte par lequel elles s'engageaient à n'opérer que dans les ZEA légalement instituées. ${ }^{29}$

Bien sûr, il peut être considéré qu'en obtenant cet engagement des coopératives, le Gouvernement a préparé les conditions pour la mise en œuvre de la nouvelle obligation imposée aux exploitants individuels. Mais il sera montré plus loin que l'Etat doit encore créer des ZEA dans lesquelles les coopératives vont opérer.

Il y a lieu également de regretter que les exploitants artisanaux individuels, principaux concernés par l'obligation, n'aient pas été associés à la démarche, alors que la nouvelle obligation leur imposée n'est pas sans poser quelque difficulté en ce qui concerne sa légalité, comme démontré dans le point qui suit.

b. Le regroupement des exploitants artisanaux comme préalable pour l'exploitation artisanale

Dans son arrêté ministériel n706/CAB.MIN/MINES/01/2010 du 20 septembre 2010 portant mesures d'encadrement de 1'Arrêté ministériel nº705/CAB.MIN/MINES/01/2010, le Ministre national des Mines subordonna la levée de la suspension de l'exploitation artisanale des minerais à l'accomplissement de plusieurs formalités, dont les plus pertinentes en rapport avec le sujet examiné ici seront analysées après avoir montré que les coopératives minières peuvent jouer un rôle bénéfique dans la lutte contre l'exploitation illégale des minerais dans l'Est du pays.

aa. L'utilité des coopératives minières dans la lutte contre l'exploitation illégale des ressources minières

Aux termes de l'article 116 du Code minier, les exploitants artisanaux opérant de manière individuelle sont tenus de vendre leur production aux négociants, aux marchés boursiers, aux comptoirs ou organismes agréés ou créés par l'Etat, ainsi qu'aux artistes agréés. Ce qui multiplie les intermédiaires commerciaux possibles et peut rendre difficile le contrôle de la production réalisée par ces exploitants artisanaux individuels.

En revanche, le groupement d'exploitants artisanaux peut permettre une amélioration du rendement des mines grâce à l'exploitation semi-industrielle et à la canalisation de toute la production réalisée (par la coopérative et ses membres) vers un point de vente. En outre,

28 NGUYEN (Quoc Dinh) et al., Droit international public, Paris, L.G.D.J., 8 édition, 2009, p.530.

29 Ministère des Mines de la RDC, Acte d'engagement solennel des coopératives minières des Provinces du Maniema, du Nord-Kivu et du Sud-Kivu, http://mines-rdc.cd/fr/documents/acte_cooperat ives.pdf, consulté le 05/08/2013. 
la coopérative pouvant elle-même procéder à l'exportation de ses produits (article 85, §2 du Code minier), elle peut obtenir des prix de vente meilleurs que ceux qu'obtiendraient des exploitants individuels localement. En plus, en mettant en place des mécanismes de collaboration adéquats avec les coopératives minières, l'Etat peut avoir des données fiables sur la production d'une ZEA. Enfin, en facilitant l'identification des exploitants artisanaux qui en sont membres et en permettant le contrôle de leurs activités de la mine au point de vente, la coopérative minière peut aider à lutter contre le commerce des minerais extraits illégalement.

En conséquence, si les coopératives minières se développent au point d'incorporer la majorité des exploitants artisanaux, elles peuvent contribuer à la lutte contre le commerce des minerais extraits par des exploitants clandestins ou par des membres des groupes armés (à la condition que les coopératives minières n'introduisent pas dans leurs stocks de produits destinés à la vente des minerais extraits par des creuseurs clandestins ou par des membres des groupes armés).

La Province du Katanga offre un exemple de l'impact positif que peut représenter la collaboration entre les coopératives minières, les services publics et les entreprises minières. En effet, par contrat conclu en 2010 entre la compagnie minière dénommée Mining Mineral Resources S.P.R.L. (MMR) et la Province du Katanga, cette dernière accorda à MMR le monopole de la commercialisation des minerais provenant des quatre principaux sites d'exploitation minière artisanale du district du Tanganyika au Nord-Katanga. En exécution de ce contrat, tous les exploitants artisanaux dans cette zone doivent vendre leur production à MMR qui, seule, est autorisée à exporter. En échange, MMR doit exécuter des projets sociaux. ${ }^{30}$

Ce contrat a fait l'objet de critiques en ce qui concerne la compétence de la Province pour accorder un tel monopole au regard du Code minier, la réalisation effective des projets sociaux par MMR, l'effet que le monopole a sur les prix des minerais achetés auprès des coopératives minières et enfin la tendance manifestée par la MMR de se faire assister dans ses activités par des agents de l'armée. ${ }^{31}$ Cependant, il a eu pour effet d'améliorer l'information des services publics sur la production réalisée par les coopératives minières partenaires de MMR (les agents de ces services étant présents sur les lieux de production et de vente) et d'écarter du commerce les minerais qui ne seraient pas vendus à MMR par lesdites coopératives. Ce qui a convaincu des sociétés comme Motorola et AVX Corporation à acheter les minerais exportés par MMR. ${ }^{32}$

31 Idem, p.21.

32 International Peace Information Service, Center for International Forestry Research, The formalisation of artisanal mining in the Democratic Republic of the Congo and Rwanda, December 2012, p.16. 
La coopérative minière: instrument de l'ingérence étatique dans la liberté d'association des exploitants miniers artisanaux en République Démocratique du Congo?

Cependant, malgré les avantages qu'offre la coopérative minière aux exploitants artisanaux, l'obligation qui a été faite à ces derniers de se regrouper mérite d'être examinée à la lumière des dispositions pertinentes de la Constitution et du Code minier.

bb. L'obligation de former des coopératives minières par rapport aux dispositions de la Constitution et du Code minier

Plusieurs formalités exigées par le Ministre national des Mines devaient être accomplies avant la reprise de l'exploitation artisanale dans les trois Provinces concernées par la mesure de suspension. ${ }^{33} \mathrm{~A}$ cet effet, quatre préalables étaient à exécuter par les services publics. Ils devaient se déployer sur le terrain pour identifier et contrôler (y compris sur le plan comptable et fiscal) tous les exploitants miniers artisanaux et tous ceux qui font le commerce des minerais, renforcer les mesures de lutte contre la fraude et la contrebande minières, organiser un fonds pour la réalisation d'infrastructures de base et enfin veiller à ce que les comptoirs d'achat des matières premières prennent des dispositions qui s'imposent pour leur transformation en entité de traitement.

En ce qui concerne les exploitants artisanaux, ils devaient, dès la prise de la mesure de suspension, ${ }^{34}$ d'abord arrêter toute activité d'extraction et de vente des substances minérales; ensuite se faire identifier et obtenir la carte d'exploitant artisanal auprès du Chef de division provinciale des mines et enfin s'organiser en coopérative minière pour la poursuite des activités minières à la levée de la suspension.

Les coopératives minières, quant à elles, devaient d'une part suspendre toute activité d'exploitation minière, d'autre part regrouper et encadrer les exploitants artisanaux en leur sein.

Les obligations assignées aux exploitants miniers artisanaux et aux coopératives minières sont critiquables au regard des dispositions des articles 58 et 56 de la Constitution. En effet, l'article 58 prévoit que tous les Congolais ont le droit de jouir des richesses nationales et garantit le droit de jouissance des ressources naturelles se trouvant sur le territoire national à tous les Congolais, qu'il s'agisse des personnes physiques ou morales. En revanche, obliger les exploitants artisanaux à créer des coopératives ou à y adhérer, revient à décider que l'exploitation minière ne peut être effectuée que par des personnes morales (soit des coopératives minières, soit des sociétés industrielles).

Par ailleurs, en garantissant aux personnes physiques de nationalité congolaise les moins nanties le droit d'exploiter les minerais de manière artisanale, c'est-à-dire avec des ressources financières, techniques et matérielles réduites, le Code minier permet à ces citoyens de jouir des immenses richesses minières dont regorge le pays. A ce titre, le Code minier est conforme à l'article 58 de la Constitution.

33 Les conditions fixées par le Ministre sont contenues aux articles 1 et 2 de l'arrêté ministériel n ${ }^{\circ} 706 /$ CAB.MIN/MINES/01/2010 du 20 septembre 2010.

34 Article 5 de l'arrêté ministériel nº706/CAB.MIN/MINES/01/2010 du 20 septembre 2010. 
Nous pouvons même affirmer que le Code minier offre plus d'avantages aux Congolais par rapport aux prévisions de l'article 58 de la Constitution. En effet, le Code minier prévoit que l'exploitation minière artisanale est réservée uniquement aux personnes physiques de nationalité congolaise. ${ }^{35}$ Il est vrai que " l'artisanat minier ne permet...généralement qu'une survie économique à court terme. Néanmoins, si le sort des creuseurs paraît peu enviable, vers quelle autre échappatoire pourraient-ils bien se tourner? $»^{36}$ En conséquence, les exploitants miniers artisanaux se trouvant dans l'incapacité (soit par ignorance, soit par manque de moyens financiers) de former des coopératives seraient privés des ressources et moyens de subsistance qu'ils tirent de l'exploitation minière. Ce qui constituerait une infraction, d'après l'article 56 de la Constitution. ${ }^{37}$

D'ailleurs, même si les exploitants parvenaient à créer une coopérative, où trouveraientils les 100.000 à 2.000 .000 de dollars américains exigés par le Règlement minier pour pouvoir procéder à une exploitation à petite échelle, ${ }^{38}$ dans la mesure où l'exploitation minière artisanale est souvent pratiquée dans des zones rurales où n'existent pas d'institutions financières et dans lesquelles les risques liés notamment à l'enclavement et à l'insécurité sont de nature à dissuader lesdites institutions d'accorder des crédits aux coopératives minières?

En définitive, décider que l'exploitation artisanale est désormais réservée aux personnes morales équivaut de la part du Ministre des Mines à priver les citoyens congolais, personnes physiques, de leurs moyens de subsistance tirés de l'exploitation de leurs ressources ou richesses naturelles.

Par ailleurs, en obligeant les exploitants artisanaux à s'organiser en coopératives minières, le Ministre des Mines crée une obligation qui n'existe pas dans le Code minier. En outre, il supprime la liberté de ces exploitants d'œuvrer de manière individuelle, pourtant prévue par le Code minier. Ainsi, l'obligation imposée par le Ministre des Mines dans l'arrêté ministériel nº706/CAB.MIN/MINES/01/2010 du 20 septembre 2010 viole l'esprit et la lettre de la Constitution et du Code minier.

Or, d'une part le Code minier a été adopté par le Parlement et il ne peut pas être modifié par un acte hiérarchiquement inférieur à savoir un arrêté ministériel; d'autre part, dans un Etat de droit, l'autorité publique doit, comme les particuliers, être soumise à la loi.

Enfin, en ce qui concerne les coopératives minières déjà existantes, l'article 2 alinéa b) de l'arrêté ministériel n706/CAB.MIN/MINES/01/2010 susdit est équivoque car les termes « regrouper et encadrer les exploitants artisanaux en leur sein », mentionnés dans cet ar-

35 Article 26 du Code minier.

36 Triest (Frédéric), op.cit., p.3.

37 En effet, l'article 56 énonce ce qui suit:

« Tout acte, tout accord, toute convention, tout arrangement ou tout autre fait, qui a pour conséquence de priver la nation, les personnes physiques ou morales de tout ou partie de leurs propres moyens d'existence tirés de leurs ressources ou de leurs richesses naturelles, sans préjudice des dispositions internationales sur les crimes économiques, est érigé en infraction de pillage punie par la loi. ».

38 Voir supra note 20. 
ticle, peuvent être compris soit comme une incitation de ces coopératives à mieux encadrer les membres en faisant déjà partie; soit comme une obligation à la charge de ces coopératives d'intégrer en leur sein de nouveaux exploitants miniers.

C'est la seconde interprétation qui paraît exprimer la pensée du Ministre, si on prend en compte l'obligation faite aux exploitants artisanaux de former des coopératives minières. En effet, cette obligation implique, d'une part, que les exploitants artisanaux forment des coopératives ou adhèrent à celles existantes; d'autre part que les coopératives ne peuvent refuser d'intégrer les candidats exploitants artisanaux qui solliciteront leur adhésion.

Et pourtant, comme dit plus haut (point II.1.2), le Règlement minier se limite à exiger du groupement d'exploitants artisanaux qui sollicite son agrément comme coopérative minière de prouver avoir proposé l'adhésion aux exploitants artisanaux (article 235 alinéa f). Ainsi, le Règlement minier laisse aux exploitants artisanaux la faculté d'adhérer ou non à la coopérative; et à la coopérative la faculté de n'accueillir que les exploitants artisanaux qui auront adhéré volontairement. Ce qui implique la liberté pour la coopérative de ne pas accueillir ceux qui ne partagent pas ses objectifs.

Imposer à une coopérative d'incorporer de nouveaux exploitants artisanaux aura pour conséquence que des exploitants artisanaux ne partageant pas forcément les idées et objectifs de la coopérative concernée intégreront ladite coopérative. Le Ministre des mines force ainsi la main à la fois aux exploitants artisanaux individuels et aux coopératives. En agissant de la sorte, il va bien au-delà de ce que prévoient le Code et le Règlement miniers. Cette contrainte exercée dans l'Est du pays sur les exploitants miniers, pour les amener à se regrouper, a vocation à se généraliser à l'ensemble de la RDC, comme le prouvent les prises de position tant des autorités publiques que de leaders d'organisations non gouvernementales organisées au sein de la société civile de la RDC. ${ }^{39}$

cc. La persistance dans les débats publics de l'idée d'imposer la constitution des coopératives minières aux exploitants artisanaux

Pour montrer à quel point l'idée d'amener les exploitants miniers artisanaux à former des coopératives se répand, nous allons, à titre d'exemple, mentionner les recommandations formulées à l'issue de la Conférence sur la bonne gouvernance et la transparence dans le secteur minier en RDC, organisée à Lubumbashi dans la province du Katanga à l'initiative du Président de la République les 30 et 31 janvier 2013. A l'issue de ces travaux, les participants ont recommandé au Ministre national des mines et aux Gouverneurs des provinces de « poursuivre l'institution des ZEA en vue d'y installer les exploitants artisanaux organi-

39 On notera que la mesure de suspension de l'exploitation artisanale fut levée en mars 2011 (Arrêté ministériel $n^{\circ} 0034 / C A B . M I N / M I N E S / 01 / 2011$ du 01er mars 2011 portant levée de la mesure de suspension des activités minières dans les provinces du Maniema, du Nord-Kivu et du Sud-Kivu). 
sés en coopératives minières ». Ils ont en plus prévu fin 2014 comme l'échéance de mise en œuvre de cette recommandation. ${ }^{40}$

Nous pouvons donc comprendre que dans l'entendement des participants à ladite conférence, ce sont les exploitants artisanaux organisés en coopératives qui sont censés travailler dans les ZEA et non pas les exploitants artisanaux opérant de manière individuelle, raison pour laquelle ils proposent un délai dont on peut imaginer qu'il servirait également à permettre aux exploitants artisanaux individuels opérant dans les ZEA de créer des coopératives ou d'y adhérer.

Défendant une position similaire, la société civile congolaise a suggéré, dans les débats en cours en RDC relatifs à une éventuelle révision du Code minier, que l'article $109 \mathrm{du}$ Code minier ${ }^{41}$, traitant de l'institution des ZEA, soit modifié de la manière ci-après:

"Les exploitants artisanaux s'organisent en coopératives minières ou toute autre forme de société commerciale prévue par la législation congolaise. Les pouvoirs publics prennent des mesures incitatives afin d'encourager la création des coopératives minières.» ${ }^{42}$

La société civile a également suggéré de prévoir, dans les dispositions transitoires du nouveau Code minier à élaborer, qu'un délai de 1 an soit accordé aux exploitants artisanaux

40 Ministère des Mines de la RDC, Conférence sur la bonne gouvernance et la transparence dans le secteur minier de la République Démocratique du Congo, les 30 et 31 janvier 2013. Matrice des recommandations, Kinshasa, 01/02/2013, p.18. A noter qu'au cours de la seconde Conférence sur la bonne gouvernance et la transparence dans le secteur minier de la République Démocratique du Congo, organisée par le Gouvernement de la RDC à Goma les 24 et 25 mars 2014, la recommandation consistant à amener les exploitants minier artisanaux à constituer des coopératives minières n'a pas été remise en cause.

41 L'article 109 du Code minier actuellement en vigueur se lit comme suit:

« Lorsque les facteurs techniques et économiques qui caractérisent certains gîtes d'or, de diamant ou de toute autre substance minérale ne permettent pas d'en assurer une exploitation industrielle ou semi-industrielle, mais permettent une exploitation artisanale, de tels gîtes sont érigés, dans les limites d'une aire géographique déterminée, en zone d'exploitation artisanale. L'institution d'une zone d'exploitation artisanale est faite par voie d'Arrêté du Ministre après avis de la Direction des Mines et du Gouverneur de la province concernée. Un Périmètre minier faisant l'objet d'un titre minier en cours de validité ne peut pas être transformé en zone d'exploitation artisanale. Un tel Périmètre est expressément exclu des zones d'exploitation artisanale instituées conformément aux dispositions de ce chapitre. L'institution d'une zone d'exploitation artisanale est notifiée au Cadastre Minier qui la porte sur les cartes de retombes minières. Tant qu'une zone d'exploitation artisanale existe, aucun titre minier ne peut y être octroyé à l'exception d'un permis de recherches demandé par un groupement des exploitants artisanaux qui travaillent dans la zone. Toutefois, la Direction de Géologie peut à tout moment procéder aux travaux de prospection et de recherches dans les zones d'exploitation artisanale.

Le Règlement Minier fixe les conditions d'octroi exceptionnel du Permis de Recherches au groupement des exploitants artisanaux. ».

42 Southern Africa Resource Watch, Amendement de la société civile congolaise pour la révision du code minier. Proposition en rapport avec le droit minier, http://www.sarwatch.org/fr/content/prop osition-en-rapport-avec-le-droit-minier, consulté le 05/08/2013. 
afin de se mettre en harmonie avec la disposition proposée. ${ }^{43}$ Les propositions de la société civile révèlent qu'elle approuve l'obligation pour les exploitants artisanaux de se constituer en coopératives minières. C'est dire que l'idée d'obliger les exploitants artisanaux à se constituer en coopératives a du succès dans l'opinion publique. La constitution de coopératives minières continue à être perçue comme obligatoire pour les exploitants miniers artisanaux ou, tout au-moins, comme devant être érigée en obligation à la charge de ces exploitants. Dans ces conditions, il n'est pas sans intérêt d'analyser l'obligation de former des coopératives minières au regard de la liberté d'association, telle qu'elle est envisagée dans le PIDCP, le PIDESC et la Charte africaine.

\section{L'OBLIGATION DE SE REGROUPER EN COOPERATIVES MINIERES FACE A LA LIBERTE D'ASSOCIATION}

Le droit à la vie, le droit de ne pas être torturé ainsi que celui de ne pas subir des traitements cruels, inhumains ou dégradants, le droit de ne pas être tenu en esclavage ou servitude et le droit à la non-rétroactivité de la loi pénale figurent au titre des droits intangibles dans le PIDCP. En plus, « le droit à la vie, le droit de ne pas être torturé et le droit à la non-rétroactivité de la loi pénale...figurent dans le 'minimum humanitaire garanti' aux victimes des conflits armés internationaux et non internationaux par l'article 3 commun aux Conventions de Genève de 1949 (l'article 3 interdit aussi la prise d'otages) ». ${ }^{44}$ A côté de ces droits intangibles, figurent d'autres droits individuels " conditionnels » ou « ordinaires » qui « sont susceptibles, toujours, de non-application temporaire (les dérogations) et aussi, parfois, d'application imparfaite (les restrictions) $» .45$

La Charte africaine contient une « clause générale de limitation dans son article 27, § 2 , qui vaut pour tous les droits qu'elle énonce $»^{46}$ et prévoit à son article 10 alinéa 2 une possibilité de restriction de la liberté d'association. ${ }^{47}$

La liberté d'association ne figure donc pas parmi les droits intangibles et est susceptible de restrictions. Dans les développements qui suivent, il sera démontré que l'obligation de regroupement à la charge des exploitants artisanaux constitue une restriction à la liberté

43 Idem.

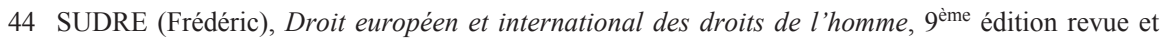
augmentée, Paris, Presses Universitaires de France, 2008, pp.207-208.

45 Idem, p.210.

46 Ibidem, p.213. L'article 27 par.2 de la Charte Africaine stipule: «Les droits et les libertés de chaque personne s'exercent dans le respect du droit d'autrui, de la sécurité collective, de la morale et de l'intérêt commun ».

47 L'article 10 de la Charte Africaine est libellé comme suit:

«1. Toute personne a le droit de constituer librement des associations avec d'autres, sous réserve de se conformer aux règles édictées par la loi.

2. Nul ne peut être obligé de faire partie d'une association sous réserve de l'obligation de solidarité prévue à l'article $29 »$. 
d'association. Ensuite, il s'agira de vérifier si cette restriction est admissible au regard des Pactes internationaux sur les droits de l'homme, de la Charte africaine et du droit interne.

\section{L'obligation de se regrouper, une ingérence de l'Etat dans le droit d'association des exploitants artisanaux}

La première question à laquelle nous allons répondre est celle de savoir si l'obligation de se regrouper en coopératives peut être considérée comme une dérogation ou plutôt une restriction à la liberté d'association des exploitants artisanaux.

L'article 4, alinéa $1^{\text {er }}$ du PIDCP précise ce qu'il faut entendre par mesure dérogatoire. Aux termes de cet article, si un danger public exceptionnel menace l'existence de la nation et est proclamé par un acte officiel, les Etats parties peuvent prendre, dans la stricte mesure où la situation l'exige, des mesures dérogeant aux obligations prévues dans le Pacte, sous réserve que ces mesures ne soient pas incompatibles avec les autres obligations que leur impose le droit international et qu'elles n'entraînent pas une discrimination fondée uniquement sur la race, la couleur, le sexe, la langue, la religion ou l'origine sociale. ${ }^{48}$ En plus, comme le précise le $\mathrm{CDH}$, « les mesures dérogeant aux dispositions du Pacte doivent avoir un caractère exceptionnel et provisoire. Avant qu'un État ne décide d'invoquer l'article 4, il faut que deux conditions essentielles soient réunies: la situation doit représenter un danger public exceptionnel qui menace l'existence de la nation et l'État partie doit avoir proclamé officiellement un état d'urgence $»{ }^{49}$

En RDC, lorsque des circonstances graves menacent, d'une manière immédiate, l'indépendance ou l'intégrité du territoire national ou qu'elles provoquent l'interruption du fonctionnement régulier des institutions, le Président de la République proclame l'état d'urgence ou l'état de siège et dans ce cas, les modalités d'application de l'état d'urgence et de l'état de siège sont déterminées par la loi (articles 85 et 86 de la Constitution). ${ }^{50}$ Dans le cas d'espèce, au moment où, en septembre 2010, le Président de la République a suspendu l'exploi-

48 Voir également GHERARI (Habib), « La Commission Africaine des Droits de l'Homme et des Peuples: bilan d'une jurisprudence ", in TAVERNIER (Paul) (dir.), Regards sur les droits de l'homme en Afrique, Paris, L'Harmattan, 2008, p.152. Cet auteur relève que, contrairement à d'autres grands textes de protection des droits de l'homme, la Charte africaine ne contient pas de clause de dérogation générale mais impose des devoirs aux individus, comme limitation aux droits proclamés. En sens contraire, voir Ouguergouz (Fatsah), La Charte africaine des droits de l'homme et des peuples, PUF, 1993, pp.257 et s., cité par SUDRE (Frédéric), op.cit., p.231, qui estime que « la Charte africaine ne contient aucune disposition spécifique; la faculté de déroger paraît alors devoir être réglée conformément au droit international général ».

49 Comité des droits de l'homme des Nations Unies, Observation générale n²9: Article 4 (Dérogations en période d'état d'urgence), HRI/GEN/1/Rev. 9 (Vol.I), p.275.

50 L'article 61 de la Constitution énumère les droits auxquels il ne peut en aucun cas être dérogé, même lorsque l'état de siège ou l'état d'urgence est proclamé: il s'agit du droit à la vie; de l'interdiction de la torture et des peines ou traitements cruels, inhumains ou dégradants; de l'interdiction de l'esclavage et de la servitude; du principe de la légalité des infractions et des peines; des droits de la défense et de recours; de l'interdiction de l'emprisonnement pour dettes; de la liberté de pen- 
tation artisanale dans l'Est du pays, il n'a pas proclamé la guerre ni l'état d'urgence ou de siège dans cette partie du pays. La mesure de suspension ne se situe donc pas dans l'hypothèse de la clause dérogatoire prévue par l'article 4 alinéa 1 du PIDCP, mais plutôt dans celle de la restriction aux droits de l'homme.

A ce stade, il est utile de signaler que dans la Constitution de la RDC, la manière dont les droits de l'homme sont formulés donne une indication sur les restrictions prévues. En ce qui concerne la liberté d'association, elle est garantie par l'article 37 qui précise que l'Etat en fixe les modalités d'exercice. ${ }^{51}$ En outre, la loi $n^{\circ} 004 / 2001$ du 20 juillet 2001 portant dispositions générales applicables aux associations sans but lucratif et aux établissements d'utilité publique précise qu'une association sans but lucratif peut être dissoute ou suspendue si elle menace la sécurité intérieure ou extérieure de l'Etat (article 53) ou si ses activités sont contraires à la loi, à l'ordre public ou aux bonnes mœurs (articles 20, 23 et 53). Ces motifs de dissolution rappellent les conditions de restriction à la liberté d'association, prévues par le PIDCP. En effet, d'après l'article 22 alinéa 2 du PIDCP, l'exercice de la liberté d'association « ne peut faire l'objet que des seules restrictions prévues par la loi et qui sont nécessaires dans une société démocratique, dans l'intérêt de la sécurité nationale, de la sûreté publique, de l'ordre public, ou pour protéger la santé ou la moralité publiques ou les droits et libertés d'autrui ». ${ }^{52}$ En conséquence, l'obligation de regroupement des exploitants artisanaux sera analysée à la lumière de ces conditions, après avoir montré en quoi ladite obligation constitue une restriction à la liberté d'association.

Avant cela, il convient de préciser que le droit d'association peut être positif ou négatif. Dans le premier cas, il consiste dans la liberté pour une personne de s'associer avec d'autres pour former une personne morale, en vue de favoriser ou protéger leurs intérêts économiques ou sociaux. La liberté d'association suppose donc « la constitution de groupes permanents ${ }^{53}$ et un « groupement volontaire et organisé de personnes physiques ou morales pour une durée plus ou moins longue en vue de la réalisation de fins communes ». ${ }^{54}$

sée, de conscience et de religion. La liberté d'association, qui ne figure pas parmi ces droits, est donc susceptible de dérogation.

51 L'article 37 de la Constitution dispose:

« L'Etat garantit la liberté d'association.

Les pouvoirs publics collaborent avec les associations qui contribuent au développement social, économique, intellectuel, moral et spirituel des populations et à l'éducation des citoyennes et des citoyens.

Cette collaboration peut revêtir la forme d'une subvention. La loi fixe les modalités d'exercice de cette liberté ».

52 De même, l'article 8 alinéa 1, a du PIDESC prévoit que l'exercice du droit de former des syndicats ou de s'y affilier ne peut faire l'objet que des seules restrictions prévues par la loi et qui constituent des mesures nécessaires, dans une société démocratique, dans l'intérêt de la sécurité nationale ou de l'ordre public, ou pour protéger les droits et les libertés d'autrui.

53 SUDRE (Frédéric), op.cit., p.550.

54 ERGEC (Rusen), Protection européenne et internationale des droits de l'homme, 2ème édition refondue, Bruxelles, Bruylant, 2006, p.241. 
Elle est prévue dans plusieurs instruments internationaux, par exemple à l'article 22 alinéa $1 \mathrm{du}$ PIDCP qui prévoit le droit pour toute personne de s'associer librement avec d'autres, y compris le droit de constituer des syndicats et d'y adhérer pour la protection de ses intérêts. Ou bien à l'article 8 alinéa 1 alinéa a du PIDESC qui énonce le droit qu'a toute personne de former avec d'autres des syndicats et de s'affilier au syndicat de son choix, sous la seule réserve fixée par l'organisation intéressée, en vue de favoriser et de protéger ses intérêts économiques et sociaux. Ou encore à l'article 10 alinéa 1 de la Charte africaine, aux termes duquel toute personne a le droit de constituer librement des associations avec d'autres, sous réserve de se conformer aux règles édictées par la loi. Enfin, l'article 37 de la Constitution de la RDC prévoit que l'Etat garantit la liberté d'association.

Pour en revenir au droit d'association positif des exploitants artisanaux, il a été démontré plus haut (point II.1.2.) que le Code et le Règlement miniers garantissent la liberté pour les exploitants artisanaux d'œuvrer de manière individuelle, ou de former des groupements. Ces deux textes sont donc compatibles avec le droit d'association positif des exploitants artisanaux. Mais le droit d'association peut aussi être négatif et signifier la liberté pour un individu de ne pas faire partie d'une association et celle pour une association de n'accepter en son sein que les individus qu'elle désire admettre, deux aspects qui méritent une attention particulière.

a. La liberté pour un individu de ne pas faire partie d'une association

Dans l'affaire Franz Wallmann et consorts c. Autriche examinée par le $\mathrm{CDH},{ }^{55}$ les auteurs de la communication, propriétaires d'un hôtel à Salzburg, accusaient l'Autriche de violation de leur liberté d'association, au motif que leur adhésion obligatoire à la Chambre régionale de commerce, de même que l'obligation de payer les cotisations annuelles à ladite Chambre, les privait de leur droit à la liberté d'association, y compris le droit de fonder ou rejoindre une autre association à des fins commerciales similaires. ${ }^{56} \mathrm{Le} \mathrm{CDH}$ considéra que l'adhésion et les cotisations obligatoires à la Chambre de commerce ne violaient pas la liberté d'association prévue à l'article 22 alinéa 1 du PIDCP parce que d'une part les Chambres de commerce sont des organismes publics créés par la loi, d'autre part l'obligation d'y adhérer et le pouvoir de faire payer des cotisations sont prévus par la loi. Enfin, le $\mathrm{CDH}$ releva que l'article $22 \mathrm{du}$ Pacte ne s'applique qu'aux associations privées et non pas aux organismes publics. ${ }^{57}$

Les coopératives minières n'étant pas des organismes publics mais plutôt des associations privées, obliger les exploitants artisanaux à y adhérer équivaut à violer la liberté d'association desdits exploitants.

55 Comité des droits de l'homme des Nations Unies, communication n ${ }^{\circ} 1002 / 2001$, Franz Wallmann et consorts c. Autriche, $C C P R / C / O P / 8$, New York and Geneva, 2007.

56 Idem, par. 3.1.

57 Ibidem, par.9.4 et 9.51. 
De manière plus explicite, l'article 10 alinéa 2 de la Charte africaine exprime l'aspect négatif de la liberté d'association dans les termes ci-après: « Nul ne peut être obligé de faire partie d'une association sous réserve de l'obligation de solidarité prévue à l'article 29 ». En application de cette disposition, la Cour africaine a précisé qu' « il y a atteinte à la liberté d'association dès lors qu'un individu est contraint de s'associer avec d'autres personnes ». ${ }^{58}$ Pour sa part, la Commission africaine a estimé que le gouvernement nigérian avait violé l'article 10 de la Charte africaine parce que d'une part il avait créé un nouvel organe (le Body of Benchers) chargé de l'administration du Barreau au Nigeria, dominé par des représentants du gouvernement et jouissant d'un grand pouvoir discrétionnaire; d'autre part il avait obligé les membres dudit Barreau à adhérer à l'organe ainsi créé. ${ }^{59}$ En plus, dans sa résolution sur le droit d'association, la Commission africaine a déclaré que les autorités législatives ne devraient pas outre passer les dispositions constitutionnelles ou faire obstacle à l'exercice des droits fondamentaux garantis par la Constitution et les normes internationales des droits de l'Homme. Elle ajouté d'une part qu'en réglementant l'usage du droit d'association, les autorités compétentes ne devraient pas décréter des mesures susceptibles d'en restreindre l'exercice et d'autre part que la restriction à l'exercice du droit à la liberté d'association devrait être compatible avec les obligations des Etats découlant de la Charte africaine des droits de l'homme et des peuples. ${ }^{60}$

En nous appuyant sur les conclusions du CDH ainsi que sur celles de la Cour et de la Commission africaines, nous pouvons conclure que l'arrêté ministériel n706/CAB.MIN/ MINES/01/2010 du 20 septembre 2010 constitue une restriction au droit d'association négatif, dans la mesure où il rend obligatoire le groupement des exploitants artisanaux et l'adhésion des ces derniers à des coopératives qui sont des personnes morales de droit privé. ${ }^{61}$

58 Cour africaine des droits de l'homme et des peuples, Requête $\mathrm{n}^{\circ} 009 / 2011$, Tanganyika Law Society et The Legal and Human Rights Centre c. République-Unie de Tanzanie; Requête nº11/2011, Christopher R. Mtikila c. République-Unie de Tanzanie, p.51, par. 113.

59 Commission africaine des droits de l'homme et des peuples, communication n ${ }^{\circ} 101 / 93$, Civil Liberties Organisations in Respect of the Nigerian Bar Association v. Nigeria, par. 14 à 17. A ce sujet, voir également HEYNS (Christof), «Civil and political rights in the African charter », in EVANS (Malcolm D.) and MURRAY (Rachel), The African Charter on Human and People's Rights. The System in Practice, 1986-2000, Cambridge University Press, 2002, p.169; ainsi que ANKUMAH (Evelyn A.), The African Commission on Human and People's Rights. Practice and Procedures, The Hague, Martinus Nijhoff Publishers, 1996, p.138.

60 Commission Africaine des Droits de l'Homme et des Peuples, Résolution $n^{\circ} 5$ sur le Droit à la Liberté d'Association, par. 1 à 3 du dispositif, http://www.achpr.org/fr/sessions/11th/resolutions/5/, consulté le 08/10/2013.

61 A titre de comparaison, l'article 11 de la Convention européenne des droits de l'homme protège aussi le droit de ne pas adhérer à une association, mais ne s'applique pas à l'obligation de s'affilier à des institutions de droit public comme les Ordres des médecins ou des avocats. Dans ce sens, voir Cour européenne des droits de l'homme, Requêtes n ${ }^{\circ} 6878 / 75$ et 7238/75, Le Compte, Van Leuven et De Meyere c. Belgique, décision du 23 juin 1981, III, par. 62-65. Voir également SUDRE (Frédéric), op.cit., p.551. Ce dernier mentionne que, « doté d'un 'effet horizontal', l'article 11 garantit non seulement le droit d'adhérer à une association mais aussi celui de ne pas adhérer ». Voir enfin ERGEC (Rusen), op.cit., p.241, lequel note que « la nature intrinsèquement vo- 
Certes, il peut être soutenu que l'Etat ne désigne pas la coopérative à laquelle adhérer et que les exploitants artisanaux sont libres dans leur choix. Il n'en demeure pas moins vrai que la possibilité de choisir n'occulte pas l'obligation de se grouper ou d'adhérer à une coopérative et que cette dernière supprime la possibilité d'opérer de manière individuelle. Par ailleurs, la liberté de choix peut être théorique dans une ZEA où il n'y a qu'une seule coopérative agréée. Dans ce dernier cas, les exploitants artisanaux individuels n'auront d'autre choix que d'adhérer à la coopérative existante. Ce qui pose la question de la liberté pour une coopérative de refuser l'adhésion de nouveaux membres.

\section{b. La liberté pour une association de refuser de nouveaux membres}

L'article 8 alinéa 1, a) du PIDESC parle du droit de toute personne de former avec d'autres des syndicats ou de s'affilier au syndicat de son choix, sous la seule réserve des règles fixées par l'organisation intéressée. ${ }^{62}$ Ainsi, comme le relève Sudre, " la liberté syndicale 'négative' est réversible, en ce sens qu'elle implique aussi le droit pour un syndicat de ne pas admettre en son sein quiconque souhaite en devenir membre et, en conséquence, de choisir librement des membres, en conformité avec les valeurs et idéaux politiques qu'il défend $\gg .63$

Par rapport à la liberté d'association, la Cour africaine a décidé que « la liberté d'association est aussi bafouée lorsque les autres citoyens sont obligés de s'associer avec un individu. En d'autres termes, la liberté d'association signifie que chacun est libre de s'associer et libre de ne pas le faire $»{ }^{64}$

Une coopérative minière a donc le droit de n'accepter comme membres que les individus qui répondent à ses exigences et objectifs. Dans ce sens, l'article 235 alinéa f du Règlement minier est compatible avec la liberté de la coopérative car, en demandant à la coopérative minière de démontrer avoir proposé l'adhésion aux exploitants artisanaux se trouvant dans la ZEA, cet article sauvegarde non seulement la liberté de ces exploitants d'adhérer à la coopérative mais aussi la liberté de la coopérative de n'admettre en son sein que les ex-

lontaire de l'association a pour effet de soustraire à l'empire de l'article 11 les associations de droit public créées par un acte unilatéral de l'autorité publique. C'est ainsi que l'obligation à charge des médecins de s'affilier à l'Ordre des médecins institué par le législateur ne constitue pas une ingérence dans la liberté d'association des médecins ").

62 La liberté de former un syndicat est prévue par l'article 38 de la Constitution de la RDC, qui dispose:

« La liberté syndicale est reconnue et garantie.

Tous les Congolais ont le droit de fonder des syndicats ou de s'y affilier librement, dans les conditions fixées par la loi ».

63 SUDRE (Frédéric), op.cit., p.557.

64 Tanganyika Law Society et The Legal and Human Rights Centre c. République-Unie de Tanzanie; Christopher R. Mtikila c. République-Unie de Tanzanie, p.51, par. 113. 
ploitants qui auront adhéré librement à ses objectifs. ${ }^{65}$ Au contraire, exiger aux coopératives minières déjà agréées d'intégrer de nouveaux exploitants artisanaux avant de reprendre leurs activités, comme le fait l'arrêté ministériel nº706/CAB.MIN/MINES/ 01/2010, équivaut non seulement à faire reculer ces coopératives en leur demandant de refaire une démarche déjà effectuée (la consultation des exploitants artisanaux), mais aussi à limiter le droit de ces coopératives de ne compter en leur sein que leurs membres actuels (qui ont adhéré librement). Ces restrictions sont-elles admissibles au regard du droit international?

2. L'obligation pour les exploitants artisanaux de se regrouper est-elle admissible au regard du droit international?

La Constitution de la RDC ne disant mot sur les motifs de restriction de la liberté d'association, c'est vers le droit international (PIDCP, PIDESC et Charte africaine) que nous allons nous tourner. Comme mentionné plus avant, les articles 22 alinéa 2 du PIDCP et 8 alinéa 1 du PIDESC précisent que l'exercice de la liberté d'association ne peut faire l'objet que des seules restrictions prévues par la loi et qui sont nécessaires dans une société démocratique, dans l'intérêt de la sécurité nationale, de la sûreté publique, de l'ordre public, ou pour protéger la santé ou la moralité publiques ou les droits et libertés d'autrui.

En ce qui concerne la Charte africaine, elle précise que « les droits et les libertés de chaque personne s'exercent dans le respect du droit d'autrui, de la sécurité collective, de la morale et de l'intérêt commun ». ${ }^{66}$ En plus, à son article 10 alinéa 2, elle stipule que «nul ne peut être obligé de faire partie d'une association sous réserve de l'obligation de solidarité prévue à l'article $29 »{ }^{67}$ L'obligation de solidarité dont mention à l'article 10 alinéa 2 cidessus se réfère, d'après la Cour africaine, à l'alinéa 4 de l'article 29 , lequel prévoit le de-

65 Cependant, l'article 235 alinéa $g$ du Règlement minier ajoute que les conditions d'adhésion à la coopérative ne doivent pas être " prohibitives». Cet article ne précise pas ce qu'il faut entendre par conditions prohibitives, ce qui risque de limiter la liberté de la coopérative dans la détermination des critères d'adhésion. A moins que, et cela devrait être indiqué dans l'article 235, par conditions prohibitives le Règlement minier ait entendu des conditions ayant un caractère discriminatoire. En effet, la Constitution de la RDC prévoit à son article 13 qu' « aucun Congolais ne peut, en matière d'éducation et d'accès aux fonctions publiques ni en aucune autre matière, faire l'objet d'une mesure discriminatoire, qu'elle résulte de la loi ou d'un acte de l'exécutif, en raison de sa religion, de son origine familiale, de sa condition sociale, de sa résidence, de ses opinions ou de ses convictions politiques, de son appartenance à une race, à une ethnie, à une tribu, à une minorité culturelle ou linguistique ». A noter aussi qu'en RDC, une association qui prône le tribalisme peut être dissoute par le Président de la République (ordonnance-loi n66-342 du 7 juin 1966).

66 Charte Africaine, article 27, par. 2.

67 L article 29 de la Charte africaine se lit comme suit:

«L'individu a en outre le devoir :

1. De préserver le développement harmonieux de la famille et d'œuvrer en faveur de la cohésion et du respect de cette famille; de respecter à tout moment ses parents, de les nourrir, et de les assister en cas de nécessité; 
voir de l'individu de préserver et de renforcer la solidarité sociale et nationale singulièrement lorsque celle-ci est menacée. ${ }^{68}$ Ainsi, pour la Cour africaine, « les Etats parties à la Charte jouissent d'une certaine mesure de discrétion concernant la limitation à la liberté d'association, dans l'intérêt de la sécurité collective, de la morale, de l'intérêt commun et qui respecte les droits et les libertés d'autrui ». ${ }^{69}$ Aussi, dans les développements qui suivent, l'obligation pour les exploitants artisanaux de se regrouper sera analysée au regard des restrictions admissibles à la liberté d'association, telles que prévues d'abord par les Pactes internationaux et ensuite par la Charte africaine. ${ }^{70}$

2. De servir sa communauté nationale en mettant ses capacités physiques et intellectuelles à son service;

3. De ne pas compromettre la sécurité de l'Etat dont il est national ou résident;

4. De préserver et de renforcer la solidarité sociale et nationale, singulièrement lorsque celle-ci est menacée;

5. De préserver et de renforcer l'indépendance nationale et l'intégrité territoriale de la patrie et, d'une façon générale, de contribuer à la défense de son pays, dans les conditions fixées par la loi;

6. De travailler, dans la mesure de ses capacités et de ses possibilités, et de s'acquitter des contributions fixées par la loi pour la sauvegarde des intérêts fondamentaux de la société;

7. De veiller, dans ses relations avec la société, à la préservation et au renforcement des valeurs culturelles africaines positives, dans un esprit de tolérance, de dialogue et de concertation et d'une façon générale de contribuer à la promotion de la santé morale de la société;

8. De contribuer au mieux de ses capacités, à tout moment et à tous les niveaux, à la promotion et à la réalisation de l'unité africaine ».

68 Tanganyika Law Society et The Legal and Human Rights Centre c. République-Unie de Tanzanie; Christopher R. Mtikila c. République-Unie de Tanzanie, pp.50-51, par. 112. Dans le sens contraire, voir HEYNS (Christof), op.cit., p.169. Ce dernier estime que même si à l'article 10 alinéa 2 l'obligation de solidarité est mentionnée au singulier, pour donner du sens à l'article 29 alinéa 4 il peut être nécessaire de se référer aux autres paragraphes, lesquels sont par conséquent indirectement visés. On notera que, comme pour l'article 10 alinéa 2, d'autres articles de la Charte africaine prévoient des limitations aux droits garantis. Ainsi l'article 8 garantit la liberté de conscience et de religion sous réserve du respect de l'ordre public; l'article 9 prévoit la liberté d'information et d'opinion dans le cadre des lois et règlements. En vertu de l'article 10 alinéa 1er toute personne a le droit de constituer librement des associations avec d'autres, sous réserve de se conformer aux règles édictées par la loi. La liberté de réunion (article 11) et celle de mouvement (article 12) sont garantis sous la réserve des restrictions nécessaires édictées par les lois et règlements, notamment dans l'intérêt de la sécurité nationale, de la sûreté d'autrui, de la santé, de la morale ou des droits et libertés des personnes. Le droit de participer au gouvernement de son pays conformément aux règles édictées par la loi est garanti à l'article 13. Il peut être porté atteinte au droit de propriété garanti par l'article 14 par nécessité publique ou dans l'intérêt général de la collectivité, conformément aux dispositions des lois appropriées. Ces limitations ont été critiquées comme étant de nature à vider de sens les droits préalablement garantis. Dans ce sens, voir ANKUMAH (Evelyn A.), op.cit., p.176.

69 Tanganyika Law Society et The Legal and Human Rights Centre c. République-Unie de Tanzanie; Christopher R. Mtikila c. République-Unie de Tanzanie, p.51, par. 112.

70 En s'appuyant sur les articles 60 et 61 de la Charte Africaine, la Commission africaine s'est inspirée, par exemple dans la communication n²28/99, Law Offices of Ghazi Suleiman v. Soudan, de la jurisprudence de la Cour Européenne et de la Cour interaméricaine des droits de l'homme. Une 
La coopérative minière: instrument de l'ingérence étatique dans la liberté d'association des exploitants miniers artisanaux en République Démocratique du Congo?

a. L'obligation de se regrouper face aux articles 22, alinéa 2 du PIDCP et 8 , alinéa 1(a) du PIDESC

Comme dit plus avant, les restrictions à la liberté d'association doivent être prévues par la loi, poursuivre un but légitime et être nécessaires dans une société démocratique, conditions dont la vérification s'impose à présent.

aa. Une restriction prévue par la loi?

L'analyse faite du Code minier et du Règlement minier a permis de démontrer que l'obligation imposée aux exploitants miniers artisanaux individuels de se regrouper n'est pas prévue par ces instruments juridiques. Cependant, le Code minier ne prévoit-il aucune disposition pour limiter l'exploitation minière? Pour y répondre, l'attention peut être focalisée sur l'article 6 dudit Code.

En vertu de l'article 6 paragraphe 1, le Président de la République peut, à son initiative ou sur proposition du Ministre, après avis du Cadastre Minier, déclarer une zone interdite aux activités minières et/ou aux travaux de carrières si la sûreté nationale, la sécurité des populations, l'incompatibilité de l'activité minière et des travaux de carrières avec d'autres usages existants ou planifiés du sol ou du sous-sol, ainsi que la protection de l'environnement l'exigent. Dans un tel cas, les droits miniers et/ou de carrières préexistant à la déclaration d'une zone interdite persistent dans la plénitude des droits qu'ils confèrent et des obligations qu'ils imposent (article 6 paragraphe 4). L'interdiction de l'activité minière prévue par l'article 6 est assimilable non pas à la restriction des droits des exploitants miniers, mais plutôt à la non-application temporaire (dérogation) de ces droits, lesquels subsistent même si leur exercice est suspendu.

Il faut relever que cet article 6 prévoit l'interdiction de l'activité minière et non pas l'imposition aux exploitants individuels d'une obligation de se regrouper. La mesure de suspension de l'exploitation artisanale aurait pu être fondée sur cet article, mais pas l'obligation de se regrouper. ${ }^{71}$ On peut dès lors se demander si cette dernière poursuit un but légitime.

approche similaire sera utilisée ici pour, le cas échéant, compléter l'analyse concernant les restrictions aux droits de l'homme par la jurisprudence de la Cour européenne.

71 Certes, le mot loi peut être compris dans un sens large et impliquer les actes réglementaires à l'instar de l'arrêté ministériel ici critiqué. Par exemple, « la 'loi matérielle' désigne donc l'ensemble du droit en vigueur, qu'il soit législatif, réglementaire ou jurisprudentiel » au sens du $\mathrm{CDH}$, comme le note SUDRE (Frédéric), op.cit., p.215. Cependant, même dans cette acception, on ne manquerait de constater, comme nous l'avons fait plus haut, que l'arrêté ministériel ne devrait pas aller à l'encontre de la loi qu'est le Code minier. 
bb. Une restriction qui vise un but légitime?

Nous avons mentionné que le but de la mesure de suspension de l'exploitation artisanale dans les trois provinces de l'Est du pays était de lutter contre l'exploitation illégale et le commerce illicite des ressources minérales, le trafic d'armes par des groupes mafieux et armés, l'insécurité récurrente dans les provinces concernées. ${ }^{72}$ Tous ces objectifs permettent de préserver la sécurité nationale, la sûreté publique et le bien-être économique du pays et sont légitimes au sens du PIDCP et du PIDESC.

L'obligation pour les exploitants artisanaux de se regrouper a été énoncée dans l'arrêté ministériel faisant suite à la suspension et en assurant l'exécution. ${ }^{73}$ Elle est fondée sur les mêmes motifs qui peuvent à première vue être considérés comme légitimes. Cependant, en examinant la politique du Ministère des Mines de la RDC, il apparaît qu'avant la décision de suspension susmentionnée, le Ministre des Mines projetait déjà d'amener les exploitants miniers artisanaux individuels à se regrouper. En effet, ledit Ministre estime que « l'exploitation artisanale a besoin...de l'organisation en coopératives ». ${ }^{74}$ Fort de ce constat, il précise son objectif qui consiste à « transformer l'exploitation minière artisanale en exploitation minière à petite échelle en passant par la mécanisation $»{ }^{75} \mathrm{~A}$ cet effet, il annonce l'action qu'il va entreprendre pour la période 2010-2015: « Organiser l'exploitation minière artisanale en coopérative et la mécaniser dans le but de favoriser l'émergence de la classe moyenne ». ${ }^{76}$ Cet objectif est constant. Il ressort également du discours du Président de la République au cours de la Conférence du 30 au 31 janvier 2013 sur la bonne gouvernance et la transparence dans le secteur minier de la RDC. A cette occasion, le Président «a convié les participants à examiner ... la rationalisation et le développement du secteur artisanal par la promotion et l'encadrement des coopératives minières $»{ }^{77}$

La politique du Gouvernement de la RDC consiste à faire évoluer les exploitants individuels artisanaux vers les coopératives minières devant procéder à une exploitation minière à petite échelle. Au vu de cette intention exprimée par l'autorité publique, il n'est pas étonnant de constater qu'en septembre 2010, l'obligation de se regrouper en coopératives ou de s'y affilier ait été imposée aux exploitants artisanaux, en même temps que l'obligation pour les coopératives minières d'intégrer les exploitants artisanaux. On peut penser que le Ministre des Mines a profité de l'occasion offerte par la suspension de l'exploitation minière

72 Voir supra, point II.2.1.

73 Arrêté ministériel n 706/CAB.MIN/MINES/01/2010 du 20 septembre 2010 portant mesures d'encadrement de l'Arrêté ministériel n 705/CAB.MIN/MINES/01/2010 du 20 septembre 2010.

74 Congomines, Vision du Ministère des Mines pour la période 2010-2015, pp.20-21, http://www.con gomines.org/wp-content/uploads/2011/10/MinistereMines-2010-Vision2010-2015.pdf\#page=21\& zoom=auto, 0,842 , consulté le $06 / 08 / 13$.

75 Idem.

76 Ibidem.

77 Ministre des Mines de la RDC, Rapport final des travaux de la Conférence sur la bonne gouvernance et la transparence dans le secteur minier de la République Démocratique du Congo, tenue à Lubumbashi, les 30 et 31 janvier 2013, Lubumbashi, $1^{\text {er }} / 02 / 2013$, p.5. 
pour exécuter son objectif planifié à l'avance. Dans cette optique, deux conclusions sont possibles. En premier lieu, l'arrêté ministériel n706/CAB.MIN/MINES/01/2010 et l'obligation pour les exploitants artisanaux individuels de se regrouper, contenue dans ledit arrêté, ont un objectif apparent (la restauration de la sécurité publique) et un objectif réel non exprimé (la transformation de l'exploitation minière artisanale en une exploitation à petite échelle). En second lieu, compte tenu de la différence entre l'objectif exprimé et l'objectif réel, l'autorité publique a exercé son pouvoir de restreindre la liberté d'association (négative) des exploitants miniers artisanaux dans un but autre que celui pour lequel la restriction a été prévue, ce qui équivaut à un détournement de pouvoir qui rend la restriction inadmissible. En effet, comme le déclare le $\mathrm{CDH}$ dans son observation générale ${ }^{\circ} 34$ sur la liberté d'opinion et la liberté d'expression, les restrictions à la liberté d'expression doivent être appliquées exclusivement aux fins pour lesquelles elles ont été prescrites et doivent être en rapport direct avec l'objectif spécifique qui les inspire. ${ }^{78}$

Dans le cas d'espèce, les buts réels visés par l'obligation de créer des coopératives ou d'y adhérer (la rationalisation et le développement de l'activité minière artisanale ainsi que la création d'une classe moyenne d'exploitants miniers congolais) sont en eux-mêmes légitimes car ils peuvent concourir au bien-être économique du pays. Ils peuvent être invoqués pour appuyer la restriction de la liberté d'association (négative) des exploitants artisanaux individuels, à condition que cela ne pas porte pas atteinte au droit des personnes physiques de nationalité congolaise de jouir des richesses naturelles du pays. En d'autres termes, on peut se demander si l'interdiction faite aux exploitants artisanaux d'opérer de manière individuelle est nécessaire et proportionnelle aux buts visés.

\section{cc. Une restriction nécessaire dans une société démocratique?}

Lles restrictions qu'un État partie impose à l'exercice de la liberté d'expression ne peuvent pas compromettre le droit lui-même et doivent répondre aux critères stricts de nécessité et de proportionnalité. ${ }^{79}$ En d'autres termes, « la règle de proportionnalité postule l'exclusivité du moyen: non seulement la limitation de la liberté doit apparaître comme le seul moyen apte à atteindre le but autorisé, mais encore, parmi plusieurs mesures qui peuvent s'offrir à elle, l'autorité doit opter pour la mesure la moins restrictive. L'autorité doit également cher-

78 Comité des droits de l'homme des Nations Unies, Observation générale $n^{\circ} 34, C C P R / C / G C / 34$, par. 4 et 22 .

79 Comité des droits de l'homme des Nations Unies, Communication $n^{\circ} 1022 / 2001$, Vladimir Velichkin v. Belarus, CCPR/C/85/D/1022/2001, par. 7. Concernant la position de la Cour européenne, voir SUDRE (Frédéric), op.cit., p.226. Ce dernier souligne que devant ladite Cour, « la notion de nécessité implique un besoin social impérieux; en particulier, la mesure prise doit être proportionnée au but légitime poursuivi... Quant à l'exigence d'un 'besoin social impérieux', la Cour ... contrôle d'abord si les motifs invoqués à l'appui des ingérences sont 'pertinents et suffisants'... Ensuite, la Cour recherche si l'ingérence est proportionnée au but légitime poursuivi et vérifie alors qu'un 'juste équilibre' a été ménagé entre l'intérêt général et les intérêts de l'individu ». 
cher à réaliser un équilibre raisonnable entre le but légitime poursuivi et les inconvénients liés à la restriction de la liberté $»{ }^{80}$

Dans le cas d'espèce, si on peut être d'accord que l'obligation imposée aux exploitants miniers artisanaux de se regrouper est fondée sur des objectifs (apparents et réels) légitimes car répondant à un besoin social impérieux, on peut cependant se demander d'abord si ladite obligation est le seul moyen permettant d'atteindre les buts visés, ensuite si elle ne porte pas atteinte à la substance même de la liberté d'association et enfin si elle maintient un équilibre raisonnable entre le but légitime poursuivi et les inconvénients liés à la restriction de la liberté d'association des exploitants artisanaux.

De l'exclusivité du moyen utilisé:

Des moyens autres que l'interdiction de l'exploitation artisanale individuelle permettent d'atteindre les objectifs (apparent et réel) du Gouvernement de la RDC. Par exemple, dans le cadre des cinquièmes journées minières de la RDC, des états généraux des mines furent organisés par le Ministre des mines à Kinshasa du 12 au 17 mars 2008. A l'issue de ces travaux, les participants adoptèrent des recommandations, parmi lesquelles la convocation d'un Groupe d'Experts chargé d'examiner les modalités de mise en place d'un Fonds Minier qui aura pour missions, entre autres, "d'accompagner l'émergence de la classe moyenne congolaise, qui devra sortir de l'artisanat minier vers la petite et moyenne entreprise, via la coopérative minière $\gg .{ }^{81}$ Cette recommandation appelle non pas à une contrainte à exercer sur les exploitants artisanaux en vue de les amener à se regrouper, mais plutôt à la prise de mesures incitatives pouvant convaincre ces exploitants qu'ils ont intérêt à se regrouper. Ce qui non seulement implique des mesures d'accompagnement, ${ }^{82}$ mais aussi laisse subsister la liberté de ceux parmi ces exploitants qui souhaiteraient continuer à opérer de manière individuelle.

De l'atteinte à la substance même de la liberté d'association:

Il a été mentionné plus haut (II.1.2.) qu'aux termes de l'article 235 alinéa f du Règlement minier, le groupement d'exploitants artisanaux qui désire obtenir des droits miniers exclusifs doit prendre la forme juridique de coopérative. En d'autres termes, dans une ZEA, les exploitants artisanaux sont libres de créer des groupements ou de continuer à opérer de manière individuelle, mais en cas de création d'un groupement, celui-ci prend la forme juridique de coopérative. Ces dispositions constituent un exemple de restriction admissible de

80 ERGEC (Rusen), op.cit., p.176.

81 Ministère des Mines de la RDC, Rapport final des états généraux des mines tenus au Centre catholique Nganda du 12 au 17 mars 2008 dans le cadre des cinquièmes journées minières de la République Démocratique du Congo, Kinshasa, avril 2008, http://miningcongo.cd/pdf/etats_gener aux_rapport_des_travaux.pdf, consulté le 10/08/2013.

82 Par exemple la formation des exploitants artisanaux à la gestion des coopératives, des facilités administratives ou même des crédits pour la création des coopératives. 
La coopérative minière: instrument de l'ingérence étatique dans la liberté d'association des exploitants miniers arti-

sanaux en République Démocratique du Congo?

la liberté d'association des exploitants artisanaux. Démonstration a également été faite (au point III.1.) que l'obligation pour les exploitants artisanaux de se regrouper ou d'adhérer à des coopératives porte atteinte à la substance même de la liberté d'une part des exploitants artisanaux d'œuvrer de manière individuelle et d'autre part des coopératives de n'admettre que les individus qu'elles souhaitent.

A ces arguments, il sied d'ajouter que le domaine minier national se présente comme suit: domaine minier concédé $=29 \%$, domaine minier réservé $=6 \%$ et $\mathrm{ZEA}=1 \% .{ }^{83}$ Les ZEA légalement instituées n'occupent donc qu'une infime portion du domaine minier du pays. ${ }^{84}$ Dans ces conditions, l'obligation de regroupement des exploitants artisanaux et même le droit à l'exploitation artisanale tout court, apparaissent comme étant dépourvus d'effectivité faute de ZEA où ces coopératives pourraient opérer. Le fait pour l'Etat de ne pas instituer suffisamment de ZEA équivaut à retirer d'une main ce qu'il a donné de l'autre, créant de ce fait un déséquilibre entre le but de l'obligation de se regrouper et ses inconvénients.

De l'équilibre entre le but poursuivi et les inconvénients liés à la restriction de la liberté:

Le regroupement des exploitants miniers artisanaux peut-être bénéfique s'il permet l'émergence d'une classe moyenne d'opérateurs miniers Congolais. Cependant, avec la suppression à terme de l'exploitation artisanale individuelle, des milliers d'exploitants artisanaux dont la survie dépend de l'activité minière se retrouveront privés de moyens de subsistance. Comme on le voit, l'obligation de constituer des coopératives comporte des inconvénients. Il s'avère ainsi nécessaire de trouver un équilibre permettant d'atteindre l'objectif poursui$\mathrm{vi}^{85}$ tout en limitant les inconvénients suscités par la restriction. Un tel équilibre peut être réalisé par exemple si l'Etat finance ou facilite le financement par les particuliers de la transformation de l'exploitation artisanale individuelle en exploitation à petite échelle. Ce qui n'est pas le cas dans la pratique car l'arrêté ministériel n706/CAB.MIN/MINES / 01/2010 du 20 septembre 2010 se contente de faire du regroupement des exploitants artisanaux ou de leur adhésion obligatoire à des coopératives un préalable à la reprise de l'exploitation artisanale, sans prévoir une assistance aux exploitants artisanaux. Il en résulte un déséquilibre parmi ces exploitants, qui profite à ceux d'entre eux qui sont nantis et qui donc

83 Congomines, op.cit., p.13. .

84 Pour la province du Sud-Kivu par exemple, Kamundala signale que sept ZEA ont été créées, à savoir les ZEA n5 à Kalehe et n¹3 à Shabunda (arrêté ministériel nº 0649/CAB.MINES/01/2009 du 22 septembre 2009), la ZEA n6254 à Kalehe (arrêté ministériel n0257/CAB.MINES/01/2008 du 13 mars 2008), la ZEA n ${ }^{\circ} 6255$ à Kalehe (arrêté ministériel n0258/CAB.MINES/01/2008 du 13 mars 2008), la ZEA n6256 à Mwenga (arrêté ministériel n0259/CAB.MINES/01/2008 du 13 mars 2008), la ZEA n6257 à Mwenga (arrêté ministériel n0260/CAB.MINES/01/2008 du 13 mars 2008) et la ZEA n ${ }^{\circ} 4$ à Shabunda (arrêté ministériel n00651/CAB.MINES/01/2009 du 24 septembre 2009); mais ces ZEA sont « difficilement localisables » et « il y a beaucoup de discordances à propos du nombre et des localisations géographiques .... L'interprétation des données du CAMI [Cadastre minier] et la confrontation de ces données entre elles-mêmes prêtent à diverses confusions ». Voir KAMUNDALA BYEMBA (Gabriel), op.cit., p.15.

85 L'émergence d'une classe moyenne congolaise. 
peuvent facilement constituer des coopératives. ${ }^{86}$ Dans ces conditions, l'Etat est loin de réaliser son objectif d'assurer l'enrichissement des exploitants artisanaux individuels et de créer une classe moyenne d'opérateurs miniers à partir desdits exploitants. En définitive, l'obligation de regroupement des exploitants artisanaux est disproportionnée par rapport au but poursuivi si des mesures adéquates d'accompagnement de ces exploitants ne sont pas prises. Cette obligation paraît également discutable au regard de la Charte africaine.

b. L'obligation de se regrouper face aux articles 21 alinéa 5, 27 alinéa 2, 29 alinéa 4 et 10 alinéa 2 de la Charte africaine

En vertu de l'article 21 alinéa 5 de la Charte africaine, l'Etat doit permettre à sa population de bénéficier pleinement des avantages provenant de ses ressources naturelles, en éliminant toutes les formes d'exploitation économique étrangère. ${ }^{87}$ Or, avec le faible pourcentage de ZEA par rapport aux concessions minières destinées à l'exploitation industrielle, l'Etat congolais privilégie l'exploitation industrielle (effectuée principalement par des entreprises étrangères) au détriment de celle artisanale. Il prive ainsi sa population de la sécurité juridique, préalable à une exploitation légale des minerais. Cela peut-être interprété comme une violation des dispositions de l'article 21 , alinéa 5 de la Charte africaine. ${ }^{88}$

En outre, l'article 27 alinéa 2 de la Charte africaine rappelle les conditions imposées par les Pactes internationaux pour qu'une restriction aux droits de l'homme soit admise, en particulier le but légitime de la restriction. Les développements faits plus haut (III.2.1.) valent donc ici aussi. D'ailleurs, pour justifier l'obligation de regroupement des exploitants artisanaux par le motif de la sécurité nationale, il faut démontrer que l'exploitation individuelle est de manière intrinsèque de nature à porter atteinte à la sécurité publique; en d'autres

86 Par exemple, pour une coopérative à créer dans la province du Sud-Kivu, l'agrément doit provenir du Ministre national des Mines à Kinshasa, ville située à plus de deux mille kilomètres du lieu où la coopérative a été créée, ce qui implique un coût financier et d'autres charges qui ne sont pas à la portée de tous les exploitants artisanaux. Ne pourrait-on pas envisager un agrément en province pour faciliter la création des coopératives minières?

87 L'article 21, alinéa 5 de la Charte africaine dispose: « les Etats parties...s'engagent à éliminer toutes les formes d'exploitation économique étrangère, notamment celle qui est pratiquée par des monopoles internationaux, afin de permettre à la population de chaque pays de bénéficier pleinement des avantages provenant de ses ressources naturelles. ».

88 L'exploitation industrielle est censée procurer des revenus plus importants à l'Etat, pouvant permettre à ce dernier, grâce à la redistribution de ces revenus, d'assurer le développement national comme le prescrit l'article 58 de la Constitution. Mais dans la réalité il n'en est pas toujours ainsi, si on tient compte du regret exprimé par les autorités publiques au cours des Journées minières organisées dans la Province du Katanga: à la fois le Président de la République et le Premier ministre ont déploré que la production minière réalisée par les nombreuses compagnies minières installées dans cette province a fortement augmenté sans que les recettes de l'Etat ne connaissent une augmentation proportionnelle. Voir Ministre des Mines de la RDC, op.cit., pp.3 et 6. En attendant que le secteur minier industriel réponde aux attentes du Gouvernement, le secteur minier artisanal assure des moyens de subsistance aux Congolais les moins nantis. Il mérite par conséquent plus d'attention de la part de l'Etat. 
termes, que le manque de sécurité n'est pas dû à d'autres facteurs comme la fragilité de l'Etat.

En ce qui concerne le devoir de solidarité prévu par les articles 10 alinéa 2 et 29 alinéa 4 de la Charte africaine, le Gouvernement congolais ne l'a pas invoqué pour justifier l'obligation de regroupement des exploitants artisanaux. ${ }^{89}$ D'ailleurs, en quoi l'exploitation minière artisanale et individuelle porte atteinte à la solidarité sociale et nationale? Et même s'il faut inclure dans le devoir de solidarité l'obligation de ne pas compromettre la sécurité de l'Etat (alinéa 3 de l'article 29), en quoi le fait d'exploiter les minerais de manière individuelle compromettrait en lui-même le contrôle la sécurité nationale? L'obligation de solidarité ne saurait donc être invoquée pour justifier l'obligation pour les exploitants artisanaux individuels de se regrouper. Quoi qu'il en soit, l'article 5 alinéa 1 du PIDCP interdit aux Etats de se livrer à un acte visant à la destruction des droits et libertés reconnus dans le Pacte ou à des limitations plus amples que celles qui y sont prévues. Enfin, la Cour africaine a précisé que les « règles et règlements ne sauraient annuler les mêmes droits et libertés qu'ils doivent régir ». ${ }^{90}$

\section{CONCLUSION}

Au vu de la pratique étatique, il s'avère que le regroupement des exploitants miniers artisanaux individuels ou l'adhésion de ces derniers à des coopératives existantes est entrain de devenir une obligation, alors que dans le Code minier actuel et dans le Règlement minier il ne s'agit que d'une faculté offerte à ces exploitants.

La présente contribution a démontré que, dans l'état actuel du droit congolais et à défaut d'accompagnement des exploitants artisanaux, cette obligation constitue une restriction inadmissible à la liberté d'association desdits exploitants car, d'une part, sur le plan interne elle n'est conforme ni à la Constitution (article 56 et 58), ni au Code miner (articles 109 et 110), ni au Règlement minier (article 235 alinéas $\mathrm{f}$ et $\mathrm{g}$ ); d'autre part, sur le plan international, elle n'est pas conforme aux exigences du PIDCP, du PIDESC et de la Charte africaine sur les restrictions à la liberté d'association, dans la mesure où elle ne satisfait pas au test de la nécessité dans une société démocratique et de la proportionnalité par rapport au but poursuivi. Enfin, les motifs invoqués par l'Etat à l'appui de ladite obligation ne sont pas conformes aux critères des exceptions prévues à l'article 29 alinéa 4 et à l'article 27 alinéa 2 de la Charte africaine, au point de justifier la limitation du droit des exploitants artisanaux de choisir de s'associer ou de ne pas s'associer.

89 En effet, comme le relève la Cour africaine dans l'affaire Tanganyika Law Society et The Legal and Human Rights Centre c. République-Unie de Tanzanie; Christopher R. Mtikila c. RépubliqueUnie de Tanzanie, p.48, par. 109, « il incombe à l'Etat de prouver qu'il est justifié de recourir aux clauses dérogatoires » de la Charte africaine.

90 Tanganyika Law Society et The Legal and Human Rights Centre c. République-Unie de Tanzanie; Christopher R. Mtikila c. République-Unie de Tanzanie, p.48, par. 109. 
En définitive, en cas de révision du Code minier, la liberté d'association des exploitants miniers artisanaux doit être maintenue, telle qu'elle est formulée dans les dispositions du Code et du Règlement miniers actuels relatives à l'exploitation artisanale des minerais, car ces dispositions protègent le double aspect positif et négatif de la liberté d'association des exploitants artisanaux. Et la pratique de l'Etat doit être en conformité avec le droit interne et le droit international.

Toutefois, notre analyse a mis en lumière des buts légitimes pouvant fonder l'obligation pour les exploitants artisanaux de se regrouper, à savoir la lutte contre la fraude des produits miniers, la lutte contre l'insécurité, l'émergence d'une classe moyenne congolaise. Aussi, une bonne façon de procéder consisterait, pour l'Etat, à poursuivre les buts légitimes ainsi revendiqués tout en tenant compte de ses obligations en matière des droits de l'homme. Parmi ces obligations, nous avons mentionné d'abord le respect des droits individuels y compris la liberté d'association dans ces deux aspects positif et négatif, ensuite la facilitation en lieu et place de l'imposition du regroupement des exploitants artisanaux individuels. ${ }^{91}$ Enfin, la réalisation du droit de la population de jouir de ses ressources naturelles exige de la part de l'Etat qu'il procède à une distribution équitable de la richesse nationale et des revenus tirés de l'exploitation des ressources naturelles, par exemple en construisant et réhabilitant des infrastructures publiques (routes, écoles, hôpitaux), en payant décemment ses agents, en organisant les services publics et en les rendant accessibles à la population. Cela contribuera à terme à créer de l'emploi, à éloigner du secteur minier artisanal ceux des Congolais qui s'y adonnent par manque d'alternative et à diminuer les pratiques négatives des services publics notamment la prolifération des taxes.

En agissant de la sorte, l'Etat pourra garantir les droits individuels et collectifs de ses nationaux. Telle est la condition pour la réalisation du but ultime poursuivi, à savoir l'émergence d'une classe moyenne de Congolais actifs dans l'ensemble de l'économie et pas seulement dans les mines. Telle est aussi l'exigence sine qua non pour pouvoir parler d'un Etat de droit en RDC, c'est-à-dire d'un Etat où règne la « prééminence du droit ».

\section{BIBLIOGRAPHIE}

\section{INSTRUMENTS JURIDIQUES INTERNATIONAUX}

- Pacte international relatif aux droits civils et politiques

- Pacte international relatif aux droits économiques, sociaux et culturels.

- Charte Africaine des Droits de l'Homme et des Peuples.

91 Au moyen de mesures incitatives comme l'institution des ZEA en nombre suffisant, l'octroi de facilités administratives ou financières aux exploitants individuels qui désirent se regrouper, l'accès au crédit pour ce faire. 
La coopérative minière: instrument de l'ingérence étatique dans la liberté d'association des exploitants miniers artisanaux en République Démocratique du Congo?

\section{INSTRUMENTS JURIDIQUES INTERNES}

- Constitution de la RDC (Journal Officiel de la RDC, $47^{\text {ème }}$ année, Numéro spécial du 18 février 2006).

- Loi n007-2002 du 11 juillet 2002 portant Code minier de la RDC (Journal Officiel de la RDC, 43ème Année, Numéro Spécial du 15 juillet 2002).

- Arrêté ministériel n 705/CAB.MIN/MINES/01/2010 du 20 septembre 2010 portant suspension des activités minières dans les provinces du Maniema, du Nord-Kivu et du SudKivu.

- Arrêté ministériel nº706/CAB.MIN/MINES/01/2010 du 20 septembre 2010 portant mesures d'encadrement de l'Arrêté ministériel n705/CAB.MIN/MINES/01/2010 du 20 septembre 2010.

- Arrêté ministériel n0034/CAB.MIN/MINES/01/2011 du 01 ${ }^{\text {er }}$ mars 2011 portant levée de la mesure de suspension des activités minières dans les provinces du Maniema, du Nord-Kivu et du Sud-Kivu.

- Décret n038/2003 du 26 mars 2003 portant Règlement minier de la RDC.

\section{DOCUMENTS OFFICIELS}

- Banque mondiale, République Démocratique du Congo. La bonne gouvernance dans le secteur minier comme facteur de croissance, Rapport $N^{\circ} 43402-Z R$, Mai 2008, $147 \mathrm{p}$.

- Comité des droits de l'homme des Nations Unies, Observation générale $n^{\circ} 34$, $C C P R / C / G C / 34$, Genève, 11-29 juillet 2011.

- Comité des droits de l'homme des Nations Unies, Observation générale n²9: Article 4, HRI/GEN/1/Rev. 9 (Vol.I), 27/05/2008, p. 275.

- Commission Africaine des Droits de l'Homme et des Peuples, Résolution $n^{\circ} 5$ sur le Droit à la Liberté d'Association.

- Journal Officiel de la République Démocratique du Congo, Instruments internationaux relatifs aux droits de l'homme ratifiés par la République Démocratique du Congo, 40 ème année, numéro spécial, 9 avril 1999, 124 p.

- Ministère des Mines de la RDC, Acte d'engagement solennel des coopératives minières des Provinces du Maniema, du Nord-Kivu et du Sud-Kivu.

- Ministère des Mines de la RDC-BGR, Rapport de l'atelier de planification du projet BGR MDM : certification des substances minérales, Bukavu, mars 2010, 62 p.

- Ministre des Mines de la RDC, Conférence sur la bonne gouvernance et la transparence dans le secteur minier de la République Démocratique du Congo, les 30 et 31 janvier 2013. Matrice des recommandations, Kinshasa, le 01/02/2013, 20 p.

- Ministère des Mines de la RDC, Rapport final des états généraux des mines tenus au Centre catholique Nganda du 12 au 17 mars 2008 dans le cadre des cinquièmes journées minières de la République Démocratique du Congo, Kinshasa, avril 2008. 
- Ministère des Mines de la RDC, Rapport final des travaux de la 2ème édition de la Conférence minière de la République démocratique du Congo élargie aux secteurs des ressources naturelles. Goma, les 24 et 25 mars 2014, 8 p.

- Ministre des Mines de la RDC, Rapport final des travaux de la Conférence sur la bonne gouvernance et la transparence dans le secteur minier de la République Démocratique du Congo, tenue à Lubumbashi, les 30 et 31 janvier 2013, Lubumbashi, le 01/02/2013, $26 \mathrm{p}$.

- Ministre des Mines de la RDC, Rapport final des travaux de la Conférence sur la bonne gouvernance et la transparence dans le secteur minier de la République Démocratique du Congo, tenue à Lubumbashi, les 30 et 31 janvier 2013, Lubumbashi, le 01/02/2013, $26 \mathrm{p}$.

- Nations Unies, Instruments internationaux relatifs aux droits de l'homme. Récapitulation des observations générales ou recommandations générales adoptées par les organes créés en vertu d'instruments internationaux relatifs aux droits de l'homme. Note du secrétariat, HRI/GEN/1/Rev. 9 (Vol.I), 27/05/2008, 312 p.

- United Nations, International covenant on civil and political rights. Selected decisions of the Human Rights Committee under the optional protocol. Volume 8. Seventy-fifth to eighty-fourth sessions (July 2002 - July 2005), CCPR/C/OP/8, New York and Geneva, 2007, 429 p.

\section{RAPPORTS D'ORGANISATIONS NON GOUVERNEMENTALES}

- Aprodeped, Rapport du séminaire de formation sur les droits des communautés locales dans l'exploitation des ressources naturelles en République Démocratique du Congo, Bukavu du 12 au 14 février 2010, 46 p.

- Global Witness, Face à un fusil, que peut- on faire? La guerre et la militarisation du secteur minier dans l'est du Congo, Rapport, juillet 2009, 115 p.

- International Peace Information Service, Center for International Forestry Research, The formalisation of artisanal mining in the Democratic Republic of the Congo and Rwanda, December 2012, $51 \mathrm{p}$.

- Ipis et International Alert, La complexité de la gestion des ressources dans un contexte de fragilité de l'Etat : une analyse du secteur minier dans l'arrière-pays du Kivu, Steven Spittaels (éd.), 2010, $72 \mathrm{p}$.

- KAMUNDALA BYEMBA (Gabriel), Exploitation minière industrielle versus exploitation minière artisanale au Sud-Kivu: Possibilités d'une cohabitation pacifique?, CEGEMI/UCB, Anvers, décembre 2012, 23 p.

- Triest (Frédéric), Justice et Paix. Analyses 2012. Le secteur minier artisanal à l'Est de la RDC : état des lieux et perspectives, Bruxelles, Commission Justice et Paix Belgique francophone, Mai 2012, $12 \mathrm{p}$. 


\section{JURISPRUDENCE}

- Comité des droits de l'homme des Nations Unies, communication n¹002/2001, Franz Wallmann et consorts c. Autriche, CCPR/C/OP/8, pp.250-259.

- Comité des droits de 1'homme des Nations Unies, communication n¹022/2001, Vladimir Velichkin v. Belarus, CCPR/C/85/D/1022/2001.

- Commission africaine des droits de l'homme et des peuples, communication n¹01/93, Civil Liberties Organisations in Respect of the Nigerian Bar Association v. Nigeria.

- Commission africaine des droits de l'homme et des peuples, communication n²24/98, Media Rights Agenda v. Nigeria.

- Commission africaine des droits de l'homme et des peuples, communication n²28/99, Law Offices of Ghazi Suleiman v. Soudan.

- Commission africaine des droits de l'homme et des peuples, communication n²68/03, Ilesanmi v. Nigéria.

- Cour africaine des droits de l'homme et des peuples, Requête n009/2011, Tanganyika Law Society et The Legal and Human Rights Centre c. République-Unie de Tanzanie; Requête $\mathrm{n}^{\circ} 011 / 2011$, Christopher R. Mtikila c. République-Unie de Tanzanie.

- Cour européenne des droits de l'homme, Requêtes nº678/75 et 7238/75, Le Compte, Van Leuven et De Meyere c. Belgique.

\section{DOCTRINE}

- ANKUMAH (Evelyn A.), The African Commission on Human and Peoples' Rights. Practice and Procedures, The Hague, Martinus Nijhoff Publishers, 1996, 246 p.

- Bakandeja Wa Mpungu (Grégoire), Droit minier et des hydrocarbures en Afrique centrale Pour une gestion rationnelle, formalisée et transparente des ressources naturelles, Bruxelles, Larcier, 2009, 344 p.

- De Failly (Didier), «Coltan : Pour comprendre... », in L'Afrique des Grands Lacs. Annuaire 2000-2001, Paris, L'Harmattan, 2001, pp.279-306.

- De Failly (Didier), «L'économie du Sud-Kivu 1990-2000 : Mutations profondes cachées par une panne ", in L'Afrique des Grands Lacs. Annuaire 1999-2000, Paris, L'Harmattan, 2000, pp.161-188.

- ERGEC (Rusen), Protection européenne et internationale des droits de l'homme, $2^{\text {ème }}$ édition refondue, Bruxelles, Bruylant, 2006, $271 \mathrm{p}$.

- GEENEN (Sara), KAMUNDALA (Gabriel) et IRAGI (Francine), « Le pari qui paralysait : La suspension des activités minières artisanales au Sud-Kivu », in L'Afrique des Grands Lacs. Annuaire 2010-2011, Paris, L'Harmattan, pp.161-183.

- GEENEN (Sara), « Constraints, Opportunities and Hope: Artisanal Gold Mining and Trade in South Kivu (DRC) », in Natural Resources and Local Livelihoods in the Greats Lakes Region of Africa, New York, Palgrave Macmillan, 2011, pp.192-212. 
- GEENEN (Sara), " Local Livelihoods, Global Interests and the State in the Congolese Mining Sector ", in Natural Resources and Local Livelihoods in the Greats Lakes Region of Africa, New York, Palgrave Macmillan, 2011, pp.149-167.

- GHERARI (Habib), « La Commission Africaine des Droits de l'Homme et des Peuples: bilan d'une jurisprudence ", in TAVERNIER (Paul) (dir.), Regards sur les droits de l'homme en Afrique, Paris, L'Harmattan, 2008, pp.132-163.

- HEYNS (Christof), "Civil and political rights in the African charter », in EVANS (Malcolm D.) and MURRAY (Rachel), The African Charter on Human and People's Rights. The System in Practice, 1986-2000, Cambridge University Press, 2002, 397 p.

- KABUYA KALALA (François) et TSHIUNZA MBIYE, « Ressources naturelles, gouvernance et défis d'une croissance soutenue en RDC », in L'Afrique des Grands Lacs. Annuaire 2008-2009, Paris, L'Harmattan, 2009, pp.141-159.

- LEPRIYA Nkoy (Arthur), « La concession mode d'exploitation minière en droit congolais », in Les analyses juridiques, $\mathrm{N}^{\circ} 4 / 2004$, Lubumbashi, 2004, pp.4-13.

- MAZALTO (Marie), Gouvernance du secteur minier et enjeux de développement en République Démocratique du Congo, Thèse de doctorat en sociologie, Montréal, Université du Québec, 2010, 530 p.

- NGUYEN (Quoc Dinh) et al., Droit international public, Paris, L.G.D.J., $8^{\mathrm{e}}$ édition, 2009, 1709 p.

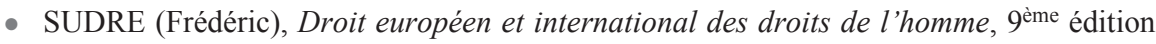
revue et augmentée, Paris, Presses Universitaires de France, 2008, 843 p.

- ZONGWE (Dunia P.), «The Legal Justifications for a People-Based Approach to the Control of Mineral Resources in the Democratic Republic of the Congo », in Cornell Law School Inter-University Graduate Student Conference Papers, Paper 12, 2008, pp. $1-78$.

\section{DOCUMENTS CONSULTES SUR INTERNET}

- Congomines, Vision du Ministère des Mines pour la période 2010-2015.

- DRC Mining ban announced by President Kabila: Impact on iTSCi Project.

- Ministère des Mines de la RDC, Carte de retombe minière de la République Démocratique du Congo.

- Pole Institute, Réouverture des activités minières à l'Est de la RD Congo : enjeux et défis, Goma, Avril 2011.

- Southern Africa Resource Watch, Amendement de la société civile congolaise pour la révision du code minier. Proposition en rapport avec le droit minier.

- Union Africaine, Liste des pays qui ont signé, ratifiéladhéré. Charte africaine des droits de l'homme et des peuples, 04/02/2010. 